\title{
An online sequential extreme learning machine for tidal prediction based on improved Gath-Geva fuzzy segmentation
}

\author{
Jianchuan Yin ${ }^{\mathrm{a}}$, Nini Wang ${ }^{\mathrm{b}, \mathrm{c}}$ \\ a Navigation College, Dalian Maritime University, 116026 Dalian, China \\ ${ }^{\mathrm{b}}$ Institute of Geographic Science and Natural Resources Research, Chinese Academy of Sciences, 100101 Beijing, China \\ c Department of Mathematics, Dalian Maritime University, 116026 Dalian, China
}

\section{A R T I C L E I N F O}

Article history:

Received 30 September 2014

Received in revised form 20 February 2015

Accepted 25 February 2015

Available online 10 August 2015

Keywords:

Online sequential extreme learning

machine

Gath-Geva fuzzy segmentation

Tidal prediction

Modular prediction

\begin{abstract}
A B S T R A C T
A novel sampling pool selection scheme is proposed for the online sequential extreme learning machine (OS-ELM) based on improved Gath-Geva (IGG) fuzzy segmentation algorithm. Tidal change is a timevarying process whose dynamics vary with changes of internal and environmental factors such as celestial bodies movements, coastal topology and environmental disturbances. When OS-ELM is implemented for identifying time-varying system dynamics, it usually sequentially selects samples with fixed number. Under such circumstance, samples representing different system dynamics are mixed together so that the online representation and prediction abilities of OS-ELM may be deteriorated. To consciously select samples with most representing ability and construct appropriate sampling pool for OS-ELM, in this study, a dynamic sampling pool selection scheme is proposed based on IGG fuzzy segmentation approach. Time series of input and output variables are segmented as per their dynamics characteristics. The change points split up the time series into several segments and the change points themselves represent the changes of system dynamics. Samples within the same segment are considered as possessing homogeneous characteristics. To achieve best representing abilities for current system dynamics, the proposed IGG-based sampling scheme is implemented for selecting sampling pool. The OS-ELM selects homogeneous samples from sampling pool thus possesses better representing ability for current dynamics. In the meantime, conventional harmonic analysis is also applied to represent the influences of celestial bodies and coastal topology. The harmonic method and IGG-based OS-ELM are combined together and the resulted modular prediction scheme is applied for online tidal level prediction of ports of King Point, Mokuoloe and Old Port Tampa in the United States. Simulation results demonstrate the feasibility and effectiveness of the proposed sampling scheme and the modular tidal prediction approach.
\end{abstract}

(c) 2015 Elsevier B.V. All rights reserved.

\section{Introduction}

Tidal prediction is an important issue in areas of oceanographic engineering, coastal engineering, tidal energy utilization as well as marine safety and efficiency [1]. Precise tidal prediction is a vital issue for navigational safety, especially when ships navigate through shallow waters or under bridges. Under both conditions the accurate prediction of tidal level is necessary for calculating water depth under keel or air clearance over ship masts. Among various tidal prediction approaches, the conventional harmonic analysis method is the most commonly used one and it is still the basis for long-term tidal prediction [2]. Harmonic analysis method expresses the tide as superposition of several sinusoidal constituents. It represents the influences on tide caused by celestial bodies and coastal topography. However, changes of tidal level are not only influenced by celestial bodies and coastal topography, but also by meteorological factors such as atmospheric pressure, wind, ice and rainfall [3]. These factors are both nonlinear and time-varying in nature, thus their influences on tidal change are complex and hard to be represented by strictly founded model. Therefore, to generate precise tidal predictions in real time, there is a practical need to construct an adaptive model to represent the influences of the above-mentioned time-varying environmental changes.

The booming intelligent computation techniques, such as neural network, fuzzy inference and evolutionary computation, have been widely applied in coastal and marine engineering industry [4]. Among those intelligent techniques, artificial neural network (ANN) can learn and represent complex nonlinear mapping underlying measured data adaptively, and stores the knowledge within computational neurons and connecting weights [5]. ANN has also been implemented in prediction of tides attributing to its natures such as inherent nonlinearity, 
universal approximation capability and parallel information processing mechanism [3,6,7].

However, the conventionally used batch learning strategy of neural network makes use of all the received samples for training, thus the achieved neural network can only represent holistic dynamics but not the time-varying dynamics of object systems. Therefore, to represent the current dynamics of time-varying system, adaptive learning strategies are needed. This leads to a novel sampling and learning scheme referred to as sequential learning scheme. Sequential learning is an adaptive learning strategy which processes samples sequentially and tunes network accordingly $[8,9]$. Sequential learning strategy is originated by resource allocation network (RAN) which learns samples one by one [8]. RAN has been developed extensively and its derivations have been implemented widely [10]. Yin et al. employ a real-time updated sliding data window (SDW) [11] which has been implemented in areas of signal processing [12] and control [13], for representing the variable system dynamics and constructs adaptive network based on learning of samples in the SDW. However, the window width is fixed and cannot adapt to the changes of system dynamics. Another type of sequential learning mode is online sequential extreme learning machine (OS-ELM) [14], which is capable of handling samples that arrive one by one or chunk by chunk. OS-ELM is derived from the novel theory of extreme learning machine (ELM) proposed by Huang et al. [15]. ELM is featured by its extremely fast learning speed and generalization capability, and its performance has been evaluated on a number of benchmark problems [16]. OS-ELM improved ELM from batch learning to be able to handle data which arrives one-by-one or chunk-by-chunk with varying chunk size [14]. OS-ELM has been applied in various areas and simulation results indicate that it produces satisfying generalization performance as well as faster training speed [14]. To improve the representation and generalization abilities of OS-ELM, there is a need for an optimal sample selection strategy to choose samples which have most representing ability for current system dynamics.

Technology of time series segmentation is employed in this study for the sample selection. Time series segmentation is a change point detection problem that can also be considered as jump analysis of a time series. Segmentation of a time series partitions a given time series into several subseries with statistical characteristics so that each segment is homogeneous, while contiguous segments are heterogeneous [17]. Based on Hubert's work [18] about the offline time series segmentation, the dynamic programming (DP) algorithm has been presented in [19]; early versions of the developed branch-and-bound (BB) approach-based algorithm appeared in [20-22]. The modified DP (mDP) algorithm [23] is a new version of the BB algorithm modified by the remaining cost concept of the DP algorithm. The BB, DP and mDP algorithms have been evaluated on several real-world hydrometeorological time series [24]. Ref. [25] proposed the modified Gath-Geva clustering algorithm for fuzzy segmentation of multivariate time series. The algorithm uses local probabilistic principal component analysis (PPCA) models to measure the homogeneity of the segments and fuzzy sets to represent segments in time, which is able to detect changes in the hidden structure of multivariate time-series [25-27].

Based on [25], the improved Gath-Geva (IGG) clustering algorithm is proposed for automatic fuzzy segmentation of univariate and multivariate time series [28]. The algorithm considers time series segmentation problem as Gath-Geva clustering and the minimum message length criterion [29] is used as segmentation order selection criterion. One characteristic of the IGG algorithm is its unsupervised nature which can automatically determine the optimal segmentation order. Another characteristic is the employment of the modified component-wise expectation maximization algorithm [30] which can avoid the sensitivity to initialization and the need to avoid the boundary of the parameter space. Other characteristic is the improvement of numerical stability by integrating segmentation order selection into model parameter estimation procedure. The proposed algorithm has been experimentally tested on artificial and real world time series and the experimental results show the effectiveness of our proposed algorithm.

In this paper, the IGG is employed as sample selection method for OS-ELM. The OS-ELM based on IGG segmentation is implemented in tidal online prediction for representing the time-varying tidal changes caused by environmental factors. In the meantime, conventional harmonic method is implemented to represent influences of celestial movements and coastal topography simultaneously. The parametric harmonic method and nonparametric OS-ELM are then combined together and the resulted modular structure can achieve better prediction accuracy and satisfy stability [31]. The OS-ELM in modular model is adjusted adaptively based on samples decided by IGG segmentation and the resulted OS-ELM is used to generate predictions sequentially at each step. Finally, the proposed modular prediction model was applied to real-time tidal level prediction at tidal gauges of several American coastal ports to validate its feasibility and effectiveness.

The remainder of this paper is organized as follows. In Section 2, we give a review on OS-ELM algorithm. In Section 3, a review is made on GG clustering-based time series segmentation algorithm and the IGG algorithm is introduced. In Section 4, the OS-ELMbased modular prediction system and the online tidal prediction simulation are presented and the performance is discussed. Conclusions are finally derived in Section 5.

\section{Online sequential extreme learning machine (OS-ELM)}

\subsection{Single hidden layer feedforward neural networks (SLFNs)}

Assume that there are $N$ arbitrary distinct samples in sampling pool $\left(\mathbf{x}_{k}, \mathbf{t}_{k}\right) \in \mathbf{R}^{n} \times \mathbf{R}^{m}$, where $\mathbf{x}_{k}$ is an input vector and $\mathbf{t}_{k}$ is the corresponding desired output. A standard single hidden layer feedforward networks (SLFNs) with $\tilde{N}$ additive hidden nodes and activation function $G(\mathbf{x})$ can be represented by

$f_{\tilde{N}}\left(\mathbf{x}_{k}\right)=\sum_{i=1}^{\tilde{N}} \boldsymbol{\omega}_{i} G\left(\mathbf{a}_{i} \cdot \mathbf{x}_{k}+b_{i}\right), \quad k=1, \ldots, N$.

where $\mathbf{a}_{i}=\left[a_{1 i}, \ldots, a_{n i}\right]^{T}$ is the weight vector connecting the input layer to the $i$-th hidden node, $b_{i}$ is the bias of the $i$-th hidden node, and $\mathbf{a}_{i} \cdot \mathbf{x}_{k}$ denotes the inner product of vectors $\mathbf{a}_{i}$ and $\mathbf{x}_{k}$ in $\mathbf{R}^{n}$. The activation functions $G(\mathbf{x})$ are Sigmoidal functions. For notational simplicity, the scalar output case is considered here. Extension to the multi-output case is straightforward. In fact, multi-output SLFNs can always be separated into a group of single output SLFNs. A schematic of the SLFNs with the scalar output is depicted in Fig. 1.

The ultimate purpose of SLFNs is to find out the values of $\omega_{i}, \mathbf{a}_{i}$ and $b_{i}$ such that $\sum_{k=1}^{N}\left\|f_{\tilde{N}}\left(\mathbf{x}_{k}\right)-t_{k}\right\|=0$, or

$f_{\tilde{N}}\left(\mathbf{x}_{k}\right)=\sum_{i=1}^{\tilde{N}} \omega_{i} G\left(\mathbf{a}_{i} \cdot \mathbf{x}_{k}+b_{i}\right)=t_{k}, \quad k=1, \ldots, N$.

Then, Eq. (2) can be written compactly as

$\mathbf{H} \omega=\mathbf{T}$,

where

$\mathbf{H}\left(\mathbf{a}_{1}, \ldots, \mathbf{a}_{\tilde{N}}, b_{1}, \ldots, b_{\tilde{N}}, \mathbf{x}_{1}, \ldots, \mathbf{x}_{N}\right)$ 


$$
\begin{gathered}
=\left[\begin{array}{ccc}
G\left(\mathbf{a}_{1} \cdot \mathbf{x}_{1}+b_{1}\right) & \cdots & G\left(\mathbf{a}_{\tilde{N}} \cdot \mathbf{x}_{1}+b_{\tilde{N}}\right) \\
\vdots & \cdots & \vdots \\
G\left(\mathbf{a}_{1} \cdot \mathbf{x}_{N}+b_{1}\right) & \cdots & G\left(\mathbf{a}_{\tilde{N}} \cdot \mathbf{x}_{N}+b_{\tilde{N}}\right)
\end{array}\right]_{N \times \tilde{N}}, \\
\boldsymbol{\omega}=\left[\begin{array}{c}
\omega_{1} \\
\vdots \\
\omega_{\tilde{N}}
\end{array}\right] \text { and } \quad \mathbf{T}=\left[\begin{array}{c}
t_{1} \\
\vdots \\
t_{N}
\end{array}\right] .
\end{gathered}
$$

where $\mathbf{H}$ is called the hidden layer output matrix of the network, the $i$-th column of $\mathbf{H}$ is the $i$-th hidden node's output vector with respect to inputs $\mathbf{x}_{1}, \mathbf{x}_{2}, \ldots, \mathbf{x}_{N}$ and the $k$-th row of $\mathbf{H}$ is the output vector of the hidden layer with respect to input $\mathbf{x}_{k}$.

\subsection{Extreme learning machine (ELM)}

In this part, we skip the rigorous proof for ELM [15,32,33]. The main idea of ELM is that for $N$ arbitrary distinct samples $\left(\mathbf{x}_{k}, t_{k}\right)$, in order to obtain arbitrarily small non-zero training error, one may randomly generate $\tilde{N}(\leq N)$ hidden nodes (with random parameters $\mathbf{a}_{i}$ and $b_{i}$ ). Under this assumption, $\mathbf{H}$ is completely defined. Then, Eq. (3) becomes a linear mapping and the output weights $\omega$ are estimated as

$\hat{\boldsymbol{\omega}}=\mathbf{H}^{\dagger} \mathbf{T}=\left(\mathbf{H}^{\mathrm{T}} \mathbf{H}\right)^{-1} \mathbf{H}^{\mathrm{T}} \mathbf{T}$,

where $\mathbf{H}^{\dagger}$ is the Moore-Penrose generalized inverse of the hidden layer output matrix $\mathbf{H}$. Calculation of the output weights can be done in a single step. This avoids any lengthy training procedure to choose control parameters (learning rate and learning epochs, etc.), thus enables its extreme processing speed. Universal approximation capability of ELM has been analyzed in [15], which indicated that SLFNs with randomly generated additive or radial basis function (RBF) nodes can universally approximate any continuous target function on any compact subspace of $\mathbf{R}_{n}$. Besides, in the implementations of ELM, the activation functions for additive nodes can be any bounded nonconstant piecewise continuous functions and the activation functions for nodes can be any integrable piecewise continuous functions.

ELM algorithm: Given a training set $\aleph=\left\{\left(\mathbf{x}_{k}, t_{k}\right) \mid \mathbf{x}_{k} \in \mathbf{R}^{n}, t_{k} \in\right.$ $R, k=1, \ldots, N\}$, activation function $G$, and hidden node number $\tilde{N}$.

Step Randomly assign hidden node parameters $\left(\mathbf{a}_{i}, b_{i}\right), i=1, \ldots$, 1: $\tilde{N}$.

Step Calculate the hidden layer output matrix $\mathbf{H}$. 2:

Step Calculate the output weight $\tilde{\boldsymbol{\omega}}: \tilde{\boldsymbol{\omega}}=\mathbf{H}^{\dagger} \mathbf{T}$. 3:

\subsection{Principle of online sequential extreme learning machine (OS-ELM)}

As training data may be presented one-by-one or chunk-bychunk in real-time applications, the ELM is modified so as to make it suitable for online sequential computation [14]. Suppose a new chunk of data is given, it results in a problem of minimizing

$$
\left\|\left[\begin{array}{l}
\mathbf{H}_{0} \\
\mathbf{H}_{1}
\end{array}\right] \omega-\left[\begin{array}{l}
\mathbf{T}_{0} \\
\mathbf{T}_{1}
\end{array}\right]\right\| .
$$

When a new sample arrives or a chunk of samples arrive, the connecting weight $\omega$ becomes

$$
\omega^{(1)}=\mathbf{K}_{1}^{-1}\left[\begin{array}{l}
\mathbf{H}_{0} \\
\mathbf{H}_{1}
\end{array}\right]^{\mathrm{T}}\left[\begin{array}{l}
\mathbf{T}_{0} \\
\mathbf{T}_{1}
\end{array}\right],
$$

where

$$
\mathbf{K}_{1}=\left[\begin{array}{l}
\mathbf{H}_{0} \\
\mathbf{H}_{1}
\end{array}\right]^{\mathrm{T}}\left[\begin{array}{l}
\mathbf{H}_{0} \\
\mathbf{H}_{1}
\end{array}\right]=\mathbf{K}_{0}+\mathbf{H}_{1}^{\mathrm{T}} \mathbf{H}_{1} .
$$

For the efficiency of sequential learning, it is reasonable to express $\omega^{(1)}$ as a function of $\omega^{(0)}, \mathbf{K}_{1}, \mathbf{H}_{1}$ and $\mathbf{T}_{1}$, which is independent of the original data set:

$$
\begin{aligned}
{\left[\begin{array}{l}
\mathbf{H}_{0} \\
\mathbf{H}_{1}
\end{array}\right]^{\mathrm{T}}\left[\begin{array}{c}
\mathbf{T}_{0} \\
\mathbf{T}_{1}
\end{array}\right] } & =\mathbf{H}_{0}^{\mathrm{T}} \mathbf{T}_{0}+\mathbf{H}_{1}^{\mathrm{T}} \mathbf{T}_{1} \\
& =\mathbf{K}_{1} \omega^{(0)}-\mathbf{H}_{1}^{\mathrm{T}} \mathbf{H}_{1} \omega^{(0)}+\mathbf{H}_{1}^{\mathrm{T}} \mathbf{T}_{1}
\end{aligned}
$$

$\omega^{(1)}$ can be expressed as follows by combining (8) and (9):

$\omega^{(1)}=\mathbf{K}_{1}^{-1}\left[\begin{array}{l}\mathbf{H}_{0} \\ \mathbf{H}_{1}\end{array}\right]^{\mathrm{T}}\left[\begin{array}{l}\mathbf{T}_{0} \\ \mathbf{T}_{1}\end{array}\right]=\omega^{(0)}+\mathbf{K}_{1}^{-1} \mathbf{H}_{1}^{\mathrm{T}}\left(\mathbf{T}_{1}-\mathbf{H}_{1} \omega^{(0)}\right)$.

Iteratively, when the $(k+1)$-th new chunk of data arrives, the recursive method is implemented for acquiring the updated solution. $\omega^{(k+1)}$ can be updated by

$\omega^{(k+1)}=\omega^{(k)}+\mathbf{K}_{k+1}^{-1} \mathbf{K}_{k+1}^{\mathrm{T}}\left(\mathbf{T}_{k+1}-\mathbf{H}_{k+1} \omega^{(k)}\right)$.

with

$\mathbf{K}_{k+1}^{-1}=\mathbf{K}_{k}^{-1}-\mathbf{K}_{k}^{-1} \mathbf{H}_{k+1}^{\mathrm{T}}\left(\mathrm{I}+\mathbf{H}_{k+1} \mathbf{K}_{k}^{-1} \mathbf{H}_{k+1}^{\mathrm{T}}\right)^{-1} \times \mathbf{H}_{k+1} \mathbf{K}_{k}^{-1}$.

\section{Improved GG clustering-based time series segmentation}

Time series segmentation is important for time series dynamics analysis and prediction. The time-series segmentation is to partition the time-series into several internally homogeneous subseries thus the samples with similar dynamics can be analyzed in one group [27]. In practice, the changes of system dynamics are not always abrupt but vague under most practical conditions, so the fuzzy clustering technique is implemented. The close relationships between fuzzy clustering techniques and probability models also indicate that it is possible to adopt probability models to modify fuzzy approaches [34]. Therefore, the probability models are also employed in this study.

\subsection{GG clustering algorithm}

The GG clustering algorithm [26] is an objective function based clustering algorithm [35]. The objective function of the GG clustering is defined as

$J_{G G}=\sum_{k=1}^{n} \sum_{i=1}^{c}\left(\mu_{i, k}\right)^{m}\left\|\mathbf{x}_{k}-\boldsymbol{\eta}_{i}^{x}\right\|^{2}=\sum_{k=1}^{n} \sum_{i=1}^{c}\left(\mu_{i, k}\right)^{m} D^{2}\left(\mathbf{x}_{k}, \boldsymbol{\eta}_{i}^{\chi}\right)$,

where $\mathbf{x}_{k}$ is a $q$-dimensional column vector, $\boldsymbol{\eta}_{i}^{\alpha}$ is the $i$-th cluster prototypes, $D^{2}\left(\mathbf{x}_{k}, \boldsymbol{\eta}_{i}^{\chi}\right)$ is the distance between the data point $\mathbf{x}_{k}$ and the $i$-th cluster center, $\mu_{i, k}$ is the membership degree of data point $\mathbf{x}_{k}$ to the $i$-th cluster and it is defined as

$\mu_{i, k}=\frac{1}{\sum_{j=1}^{c}\left(D\left(\mathbf{x}_{k}, \boldsymbol{\eta}_{i}^{\chi}\right) / D\left(\mathbf{x}_{k}, \boldsymbol{\eta}_{j}^{\chi}\right)\right)^{2 /(m-1)}}$,

$m \in(1, \infty)$ is the weighting exponent that determines the fuzziness of the resulting clusters. A common choice of the weighting exponent is $m=2$ and this value will be used throughout this paper. The membership degree $\mu_{i, k}$ is subject to the following constraints:

$\mu_{i, k} \in[0,1], \quad \forall i, k ; \quad 0<\sum_{k=1}^{n} \mu_{i, k}<n, \quad \forall i ; \quad \sum_{i=1}^{c} \mu_{i, k}=1, \quad \forall k$. 


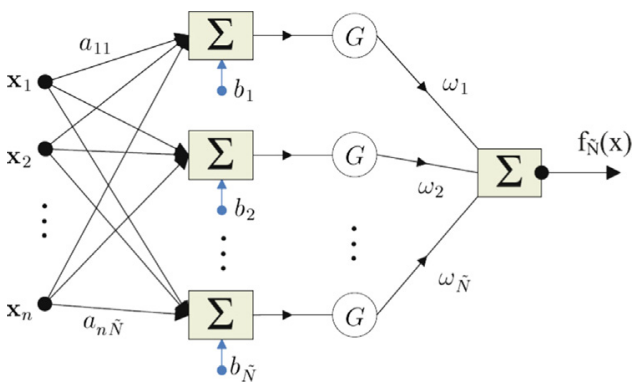

Fig. 1. The schematic of the SLFNs with the scalar output.

The distance measurement $D^{2}\left(\mathbf{x}_{k}, \boldsymbol{\eta}_{i}^{x}\right)$ is chosen proportional to the posteriori probability $p\left(\mathbf{x}_{k} \mid \boldsymbol{\eta}_{i}^{\chi}\right)$ as follows:

$D^{2}\left(\mathbf{x}_{k}, \boldsymbol{\eta}_{i}^{\chi}\right)=\frac{1}{P_{i} p\left(\mathbf{x}_{k} \mid \boldsymbol{\eta}_{i}^{\chi}\right)}$,

where $p\left(\mathbf{x}_{k} \mid \boldsymbol{\eta}_{i}^{\chi}\right)$ is the probability that $\mathbf{x}_{k}$ belongs to the $i$-th cluster, which is represented by Gaussian function $G\left(\mathbf{x}_{k} ; \mathbf{v}_{i}^{x}, \mathbf{F}_{i}^{x}\right)$ as follows:

$$
\begin{aligned}
p\left(\mathbf{x}_{k} \mid \boldsymbol{\eta}_{i}^{\chi}\right)= & G\left(\mathbf{x}_{k} ; \mathbf{v}_{i}^{x}, \mathbf{F}_{i}^{\chi}\right)=\frac{1}{(2 \pi)^{q / 2} \sqrt{\operatorname{det} \mathbf{F}_{i}^{\chi}}} \\
& \cdot \exp \left(-\frac{1}{2}\left(\mathbf{x}_{k}-\mathbf{v}_{i}^{x}\right)^{T}\left(\mathbf{F}_{i}^{\chi}\right)^{-1}\left(\mathbf{x}_{k}-\mathbf{v}_{i}^{x}\right)\right),
\end{aligned}
$$

where $\mathbf{v}_{i}^{\chi}$ and $\mathbf{F}_{i}^{\chi}$ are, respectively, centers and covariances of Gaussian function $G\left(\mathbf{x}_{k} ; \mathbf{v}_{i}^{x}, \mathbf{F}_{i}^{x}\right)$, and $P_{i}$ is the coefficient designed for eliminating the sensitivity of the algorithm, which is given by

$P_{i}=\frac{1}{n} \sum_{k=1}^{n} \mu_{i, k}$.

Assuming that $\mathbf{x}_{k}$ is effectively modeled as a mixture of Gaussian distribution, the GG clustering [26] is equivalent to the EM identification of the Gaussian mixture.

\subsection{GG clustering-based time series segmentation}

A time series $\mathcal{X}=\left\{\mathbf{x}_{k} \mid k=1, \ldots, n\right\}$ is a finite set of $n$ samples labeled by time-coordinate $\mathcal{T}=\left\{t_{k} \mid k=1, \ldots, n\right\}$, where $\mathbf{x}_{k}=\left[x_{1, k}\right.$, $\left.x_{2, k}, \ldots, x_{q, k}\right]^{T}, 1 \leq k \leq n$. Instead of defining crisp bounds of the segments, Abonyi et al. developed an algorithm for dividing time series into fuzzy segments [25,27], which considers time series segmentation as GG clustering with time-coordinate as an additional variable. Assuming that the data point $\mathbf{z}_{k}=\left[t_{k}, \mathbf{x}_{k}^{T}\right]^{T}$ can be effectively modeled as a mixture of multivariate Gaussian distribution, the objective function can be written as

$J_{G G T S}=\sum_{k=1}^{n} \sum_{i=1}^{c}\left(\mu_{i, k}\right)^{m}\left\|\mathbf{z}_{k}-\boldsymbol{\eta}_{i}\right\|^{2}=\sum_{k=1}^{n} \sum_{i=1}^{c}\left(\mu_{i, k}\right)^{m} D^{2}\left(\mathbf{z}_{k}, \boldsymbol{\eta}_{i}\right)$.

Since $t_{k}$ is independent of $\mathbf{x}_{k}$, the distance measurement of Eq. (19), $D^{2}\left(\mathbf{z}_{k}, \boldsymbol{\eta}_{i}\right)$, is defined as $[25,27]$

$D^{2}\left(\mathbf{z}_{k}, \boldsymbol{\eta}_{i}\right)=\frac{1}{\alpha_{i} p\left(\mathbf{z}_{k} \mid \boldsymbol{\eta}_{i}\right)}=\frac{1}{\alpha_{i} p\left(t_{k} \mid \boldsymbol{\eta}_{i}^{t}\right) p\left(\mathbf{x}_{k} \mid \boldsymbol{\eta}_{i}^{\alpha}\right)}$.

In Eq. (20), probability density function $p\left(t_{k} \mid \boldsymbol{\eta}_{i}^{t}\right)$ is given by Gaussian function $G\left(t_{k} ; v_{i}^{t}, \sigma_{i, t}^{2}\right)$ to represent the probability of $t_{k}$ belonging to the $i$-th cluster in time-coordinate [25,27]:

$p\left(t_{k} \mid \boldsymbol{\eta}_{i}^{t}\right)=G\left(t_{k} ; v_{i}^{t}, \sigma_{i, t}^{2}\right)=\frac{1}{\sqrt{2 \pi \sigma_{i, t}^{2}}} \exp \left(-\frac{1}{2} \frac{\left(t_{k}-v_{i}^{t}\right)^{2}}{\sigma_{i, t}^{2}}\right)$,

where $v_{i}^{t}$ and $\sigma_{i, t}^{2}$ are separately centers and variances of Gaussian function $G\left(t_{k} ; v_{i}^{t}, \sigma_{i, t}^{2}\right)$. Probability density function of Eq. (20), $p\left(\mathbf{x}_{k} \mid \boldsymbol{\eta}_{i}^{\chi}\right)$, is given by Gaussian function $G\left(\mathbf{x}_{k} ; \mathbf{v}_{i}^{x}, \mathbf{F}_{i}^{x}\right)$ to represent the probability of $\mathbf{x}_{k}$ belonging to the $i$-th cluster in time series data $[27,25]$ :

$$
\begin{aligned}
p\left(\mathbf{x}_{k} \mid \boldsymbol{\eta}_{i}^{\chi}\right)= & G\left(\mathbf{x}_{k} ; \mathbf{v}_{i}^{\chi}, \mathbf{F}_{i}^{\chi}\right)=\frac{1}{(2 \pi)^{\frac{r}{2}} \sqrt{\operatorname{det} \mathbf{F}_{i}^{\chi}}} \\
& \cdot \exp \left(-\frac{1}{2}\left(\mathbf{x}_{k}-\mathbf{v}_{i}^{\chi}\right)^{T}\left(\mathbf{F}_{i}^{\chi}\right)^{-1}\left(\mathbf{x}_{k}-\mathbf{v}_{i}^{\chi}\right)\right),
\end{aligned}
$$

where $\mathbf{v}_{i}^{x}$ and $\mathbf{F}_{i}^{x}$ are separately centers and covariances of Gaussian function $G\left(\mathbf{x}_{k} ; \mathbf{V}_{i}^{x}, \mathbf{F}_{i}^{x}\right)$, and $r$ is the rank of $\mathbf{F}_{i}^{x}$. In Eq. (20), $\alpha_{i}$ is the mixing coefficient satisfying

$\sum_{i=1}^{c} \alpha_{i}=1, \alpha_{i} \geq 0, \quad i=1, \ldots, c$.

The optimization of the parameters $\boldsymbol{\eta}=\left\{v_{i}^{t}, \sigma_{i, t}^{2}, \mathbf{v}_{i}^{x}, \mathbf{F}_{i}^{x}, \alpha_{i} \mid 1 \leq\right.$ $i \leq c\}$ is considered as the EM identification of Gaussian mixture (Eqs. (21) and (22)). The fuzzy segmentation of a time series, $\beta_{i}\left(t_{k}\right)$, is defined as $[27,25]$

$\beta_{i}\left(t_{k}\right)=\frac{A_{i}\left(t_{k}\right)}{\sum_{j=1}^{c} A_{j}\left(t_{k}\right)} \in[0,1]$,

where $A_{i}\left(t_{k}\right)$ is Gaussian membership function given by

$A_{i}\left(t_{k}\right)=\exp \left(-\frac{1}{2} \frac{\left(t_{k}-v_{i}^{t}\right)^{2}}{\sigma_{i, t}^{2}}\right)$.

\subsection{Improved GG clustering-based time series segmentation}

In this section, the GG clustering is improved for the automatic fuzzy segmentation of time series with time-varying dynamics.

A. MML criterion for GG clustering-based time series segmentation algorithm: Segmentation order selection of a time series is often facilitated by the use of the model selection criterion [19]. The model selection criterion is a tradeoff between data fitting accuracy and complexity. MML criterion is a powerful model selection criterion that has not yet been applied to solve time series segmentation problem [29].

The definition of distance measurement $D^{2}\left(\mathbf{z}_{k}, \boldsymbol{\eta}_{i}\right)$ is defined as [36]

$D^{2}\left(\mathbf{z}_{k}, \boldsymbol{\eta}_{i}\right)=-\log \alpha_{i} p\left(\mathbf{z}_{k} \mid \boldsymbol{\eta}_{i}\right)=-\log \alpha_{i} p\left(t_{k} \mid \boldsymbol{\eta}_{i}^{t}\right) p\left(\mathbf{x}_{k} \mid \boldsymbol{\eta}_{i}^{\chi}\right)$.

By substituting Eq. (26) into Eq. (19), the objective function of improved GG clustering-based time series segmentation algorithm is expressed as

$J_{I G G T S}=-\sum_{k=1}^{n} \sum_{i=1}^{c}\left(\mu_{i, k}\right)^{m} \log \alpha_{i} p\left(t_{k} \mid \boldsymbol{\eta}_{i}^{t}\right) p\left(\mathbf{x}_{k} \mid \boldsymbol{\eta}_{i}^{x}\right)$.

MML criterion for improved GGC-based time series segmentation algorithm takes the following form:

$$
\begin{aligned}
\operatorname{MesSLen~}_{\text {IGGTS }}(\boldsymbol{\eta}, \mathcal{Z})= & -\sum_{k=1}^{n} \sum_{i=1}^{c}\left(\mu_{i, k}\right)^{m} \log \alpha_{i} p\left(t_{k} \mid \boldsymbol{\eta}_{i}^{t}\right) p\left(\mathbf{x}_{k} \mid \boldsymbol{\eta}_{i}^{x}\right) \\
& +\frac{N}{2} \sum_{i=1}^{c} \log \frac{n \alpha_{i}}{12}+\frac{c}{2} \log \frac{n}{12}+\frac{c(N+1)}{2}
\end{aligned}
$$

It is stated that if any of the $\alpha_{i}$ s is zero, MML penalty term in Eq. (28) does not make sense [29]. This difficulty is solved by deleting those clusters whose probability is zero, and only coding parameters of clusters whose probability is nonzero [29]. Following [29], MML criterion for the improved GG clustering-based time series segmentation algorithm is derived from Eq. (28) as follows:

$$
\begin{aligned}
\operatorname{MesSLen~}_{\text {IGGTS }}\left(\boldsymbol{\eta}_{\text {curr }}, \mathcal{Z}\right)= & -\sum_{k=1}^{n} \sum_{i=1}^{c_{n z}}\left(\mu_{i, k}\right)^{m} \log \alpha_{i} p\left(t_{k} \mid \boldsymbol{\eta}_{i}^{t}\right) p\left(\mathbf{x}_{k} \mid \boldsymbol{\eta}_{i}^{\alpha}\right) \\
& +\frac{N}{2} \sum_{i=1}^{c_{n z}} \log \frac{n \alpha_{i}}{12}+\frac{c_{n z}}{2} \log \frac{n}{12}+\frac{c_{n z}(N+1)}{2},
\end{aligned}
$$


where $c_{n z}$ denotes the number of non-zero-probability clusters, and $\boldsymbol{\eta}_{\text {curr }}=\left\{\boldsymbol{\eta}_{i}, \alpha_{i} \mid i=1, \ldots, c_{n z}\right\}=\left\{\boldsymbol{\eta}_{i}^{t}, \boldsymbol{\eta}_{i}^{\chi}, \alpha_{i} \mid i=1, \ldots, c_{n z}\right\}$ represents the vector of the current parameters.

B. Algorithm and implementation: To couple segmentation order selection and model parameter estimation in a unified framework, a reformulation of $\mathrm{CEM}^{2}$ algorithm [29] for GG clustering-based time series segmentation is employed. The algorithm starts with a large number of clusters all over the space, which makes the algorithm robust with respect to initialization. The necessary clusters are selected in a top-down manner. Let

$$
\begin{aligned}
\boldsymbol{\eta}(l) & =\left\{\boldsymbol{\eta}_{i}(l), \alpha_{i}(l) \mid i=1, \ldots, c_{n z}\right\} \\
& =\left\{\boldsymbol{\eta}_{i}^{t}(l), \boldsymbol{\eta}_{i}^{x}(l), \alpha_{i}(l) \mid i=1, \ldots, c_{n z}\right\} \\
& =\left\{v_{i}^{t}(l), \sigma_{i, t}^{2}(l), \mathbf{v}_{i}^{x}(l), \mathbf{F}_{i}^{\chi}(l), \alpha_{i}(l) \mid i=1, \ldots, c_{n z}\right\}
\end{aligned}
$$

be the vector of parameters at the current iteration. The E-step updates the fuzzy partition matrix $\mathbf{U}(l)=\left[\mu_{i, k}^{(l)}\right]_{c_{n z} \times n}$, where $\mu_{i, k}^{(l)}$ is the membership degree of the $k$-th data point to the $i$-th cluster:

$$
\begin{aligned}
\mu_{i, k}^{(l)} & =\frac{1}{\sum_{j=1}^{c_{\text {nz }}}\left(D\left(\mathbf{z}_{k}, \boldsymbol{\eta}_{i}(l)\right) / D\left(\mathbf{z}_{k}, \boldsymbol{\eta}_{j}(l)\right)\right)^{2 / m-1}} \\
& =\frac{\left(\alpha_{i}(l) p\left(t_{k} \mid \boldsymbol{\eta}_{i}^{t}(l)\right) p\left(\mathbf{x}_{k} \mid \boldsymbol{\eta}_{i}^{\chi}(l)\right)\right)^{m-1}}{\sum_{j=1}^{c_{\text {nz }}}\left(\alpha_{j}(l) p\left(t_{k} \mid \boldsymbol{\eta}_{j}^{t}(l)\right) p\left(\mathbf{x}_{k} \mid \boldsymbol{\eta}_{j}^{\chi}(l)\right)\right)^{m-1}} .
\end{aligned}
$$

Then, the M-step involves operations of updating the vector of the parameters $\boldsymbol{\eta}(l)$ and annihilating clusters with vanishing mixing coefficients simultaneously. For the $i$-th cluster, the mixing coefficient $\alpha_{i}(l+1)$ can be calculated by $[29,37]$

$$
\alpha_{i}(l+1)=\frac{\max \left\{0, \sum_{k=1}^{n} \mu_{i, k}^{(l)}-\frac{N}{2}\right\}}{\sum_{j=1}^{c_{n z}} \max \left\{0, \sum_{k=1}^{n} \mu_{j, k}^{(l)}-\frac{N}{2}\right\}} .
$$

Consider the constraints in Eq. (23), the normalized mixing coefficient vector $\mathcal{W}(l+1)$ is defined as

$\mathcal{W}(l+1)=\left\{\alpha_{1}(l+1), \ldots, \alpha_{c_{n z}}(l+1)\right\}=\frac{\left\{\alpha_{1}(l+1), \ldots, \alpha_{c_{n z}}(l+1)\right\}}{\sum_{j=1}^{c_{n z}} \alpha_{j}(l+1)}$.

The $i$-th cluster corresponding to $\alpha_{i}(l+1)=0$ is annihilated, else the parameters of the $i$-th cluster $\boldsymbol{\eta}_{i}(l+1)$ are updated by

$$
\begin{aligned}
& v_{i}^{t}(l+1)=\left(\sum_{k=1}^{n}\left(\mu_{i, k}^{(l)}\right)^{m}\right)^{-1} \sum_{k=1}^{n}\left(\mu_{i, k}^{(l)}\right)^{m} t_{k}, \\
& \mathbf{v}_{i}^{x}(l+1)=\left(\sum_{k=1}^{n}\left(\mu_{i, k}^{(l)}\right)^{m}\right)^{-1} \sum_{k=1}^{n}\left(\mu_{i, k}^{(l)}\right)^{m} \mathbf{x}_{k}, \\
& \sigma_{i, t}^{2}(l+1)=\frac{\sum_{k=1}^{n}\left(\mu_{i, k}^{(l)}\right)^{m}\left(t_{k}-v_{i}^{t}(l+1)\right)\left(t_{k}-v_{i}^{t}(l+1)\right)^{T}}{\sum_{k=1}^{n}\left(\mu_{i, k}^{(l)}\right)^{m}},
\end{aligned}
$$

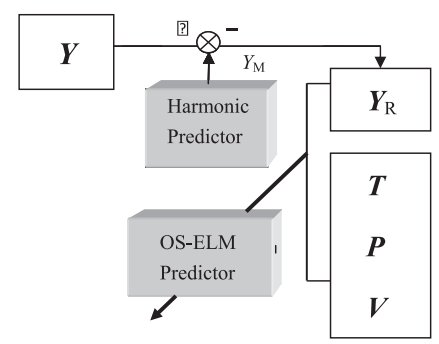

Fig. 2. Identification process of the modular model based on OS-ELM.

$$
\mathbf{F}_{i}^{\chi}(l+1)=\frac{\sum_{k=1}^{n}\left(\mu_{i, k}^{(l)}\right)^{m}\left(\mathbf{x}_{k}-\mathbf{v}_{i}^{\chi}(l+1)\right)\left(\mathbf{x}_{k}-\mathbf{v}_{i}^{\chi}(l+1)\right)^{T}}{\sum_{k=1}^{n}\left(\mu_{i, k}^{(l)}\right)^{m}} .
$$

It is noted that if the initial number of clusters $c_{n z}$ is too large, it may happen that no cluster has enough initial support $\left(\sum_{k=1}^{n}\right.$ $\mu_{i, k}^{(l)}<N / 2$ for $i=1, \ldots, c_{n z}$ ) [29]. To sidestep this difficulty, $\mathrm{CEM}^{2}$ with the $M$-step is adopted to sequentially update parameters $\boldsymbol{\eta}(l)$ at the $l$-th iteration: update $\alpha_{1}(l+1)$ by Eq. (31) and $\boldsymbol{\eta}_{1}(l+1)$ by Eq. (33), recompute $\mathcal{W}(l+1)$ by Eq. (32), update $\alpha_{2}(l+1)$ and $\boldsymbol{\eta}_{2}(l+1)$, recompute $\mathcal{W}(l+1)$, and so on.

After convergence is completed, i.e., when the relative difference between the fuzzy partition matrices of the previous and the current iteration $\|\mathbf{U}(l)-\mathbf{U}(l-1)\|$ falls below a threshold $\varepsilon$, there is no guarantee that we have found a minimum of MML criterion given by Eq. (29). Follow the method of [29], we check if smaller values of MML criterion are achieved by annihilating the least probable cluster with smallest $\alpha_{i}$.

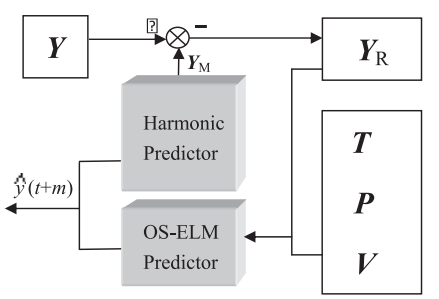

Fig. 3. Prediction process of the modular model based on OS-ELM.

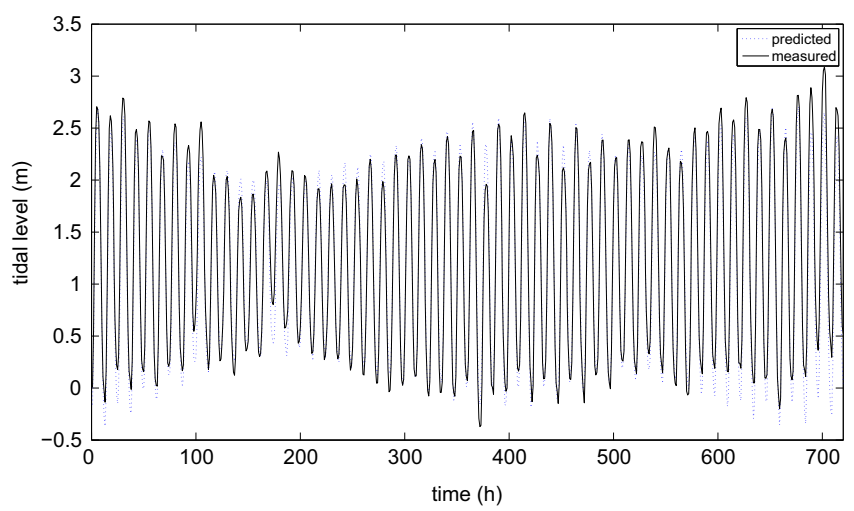

Fig. 4. Measured tidal level and the predicted results by using harmonic method (King Point).

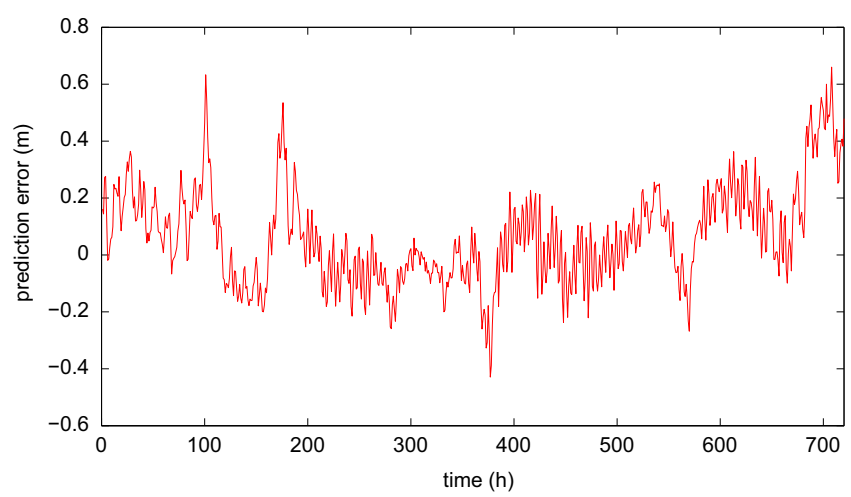

Fig. 5. Prediction error by using harmonic method (King Point). 

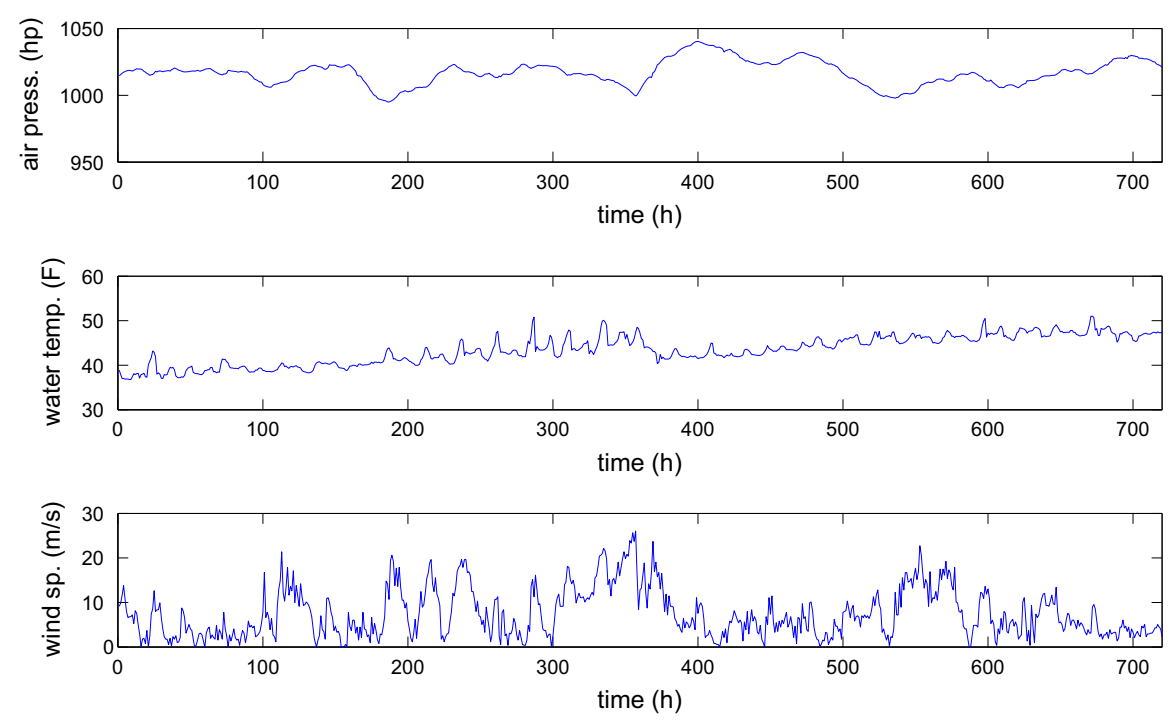

Fig. 6. Changes of environmental factors of air pressure, water temperature and wind speed (King Point).

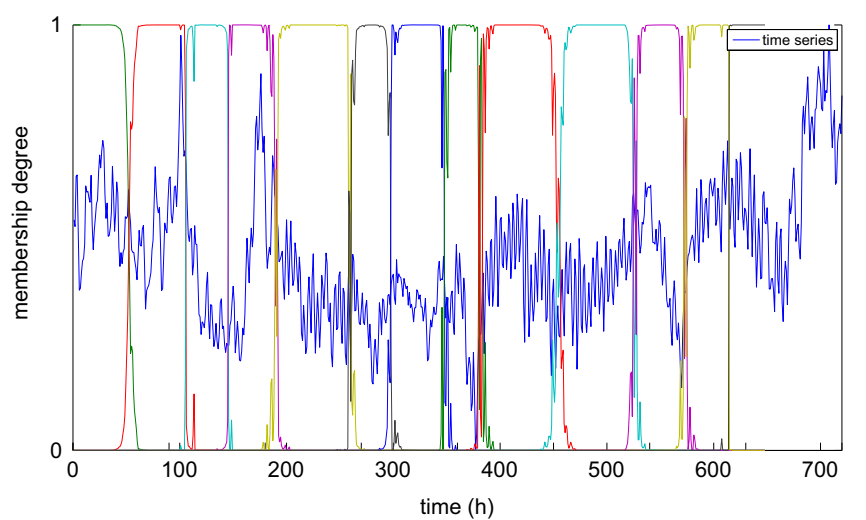

Fig. 7. Optimal membership degrees (King Point).

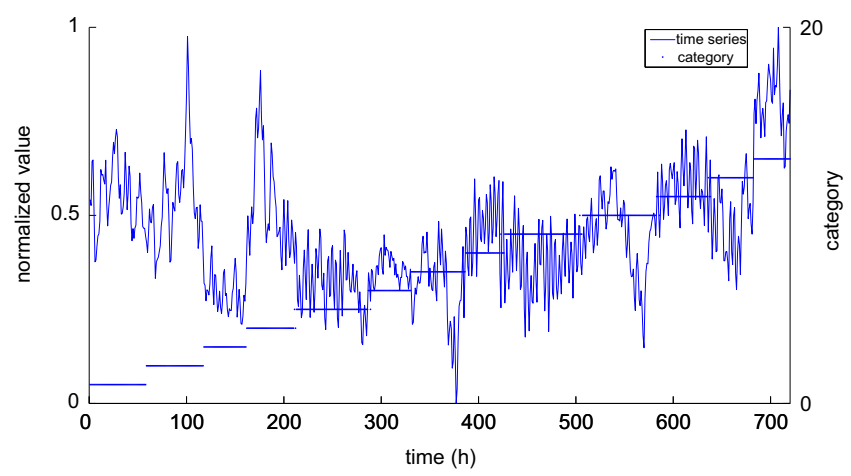

Fig. 8. Optimal crisp segmentation result based on membership degrees (King Point).

\subsection{IGG-based sampling pool selection for OS-ELM}

The IGG algorithm segments time series into separated sections. The crisp segmentation points represent the abrupt changes of system dynamics; and each segmental section represents homogeneous system dynamics. The tidal change is a complex process which is influenced by various environmental factors. Considering that different factors may exhibit different timedelays for their effects to appear, the crisp segmentation points of the tidal changes and environmental factors are not coinciding strictly. Furthermore, the changes of tidal changes and environmental changes are mostly time-varying. Therefore, to represent the current system dynamics sufficiently, the union of sections corresponding to current tidal and environmental changes is selected for representing current system dynamics.

At time $t$, the $i$-th input sample is denoted as $I_{i}(t)$ and the $j$-th output sample is denoted as $O_{j}(t)$, where $1 \leq i \leq m$ and $1 \leq j \leq n$ with $m$ and $n$ are the number of variables of input and output, respectively. The segmented sections which $I_{i}(t)$ and $O_{j}(t)$ belong to are denoted as $S_{I_{i}}(t)$ and $S_{O_{j}}(t)$, respectively.

Therefore, the union of current sections is expressed as

$\mathbf{Q}(t)=\bigcup\left(S_{I_{1}}(t), \ldots, S_{I_{i}}(t), \ldots, S_{I_{m}}(t), S_{O_{1}}(t), \ldots, S_{O_{j}}(t), \ldots, S_{O_{n}}(t)\right)$,

the union is then served as the sampling pool for OS-ELM, and the length of $Q(t)$ is denoted as $L_{\mathbf{Q}}$. At $t$-th step, after the segmentation is processed and the union is determined according to (34), OSELM is implemented for identification and prediction based on the samples in $Q(t)$.

The IGG method is conducted sequentially in this study. To ensure the processing speed of IGGf method, a sliding window is utilized to limit the computational burden of the algorithm. The sliding window is a first-in-first-out (FIFO) sequence. When a new sample is received, the new sample is added in the window and the foremost one is deleted from window. However, the window should be large enough to guarantee the stability of the algorithm. Take both consideration of processing speed and stability, the width of the sliding window is set as 200 in this study. The process of online application of IGG is stated as follows:

Step 1: The segmentation is performed initially for the received samples. In this study, the first 200 samples are segmented and there are initially $g-1$ segments corresponding to $g$ change points. After all the initial $g$ change points are fixed, the IGG is then performed to decide the new change point.

Step 2: When a new sample is received, the segmentation is conducted within the updated window by using IGG method. There are $h$ change points in the window and the $h$-th change point is the newest one.

Step 3: If the $h$-th change point falls behind the $g$-th change point, it is set as a new change point, the $(g+1)$-th change point. The determination of the new change point does not affect the previously decided change points.

Step 4: The IGG is conducted sequentially likewise and the sampling pool is decided consequently. 

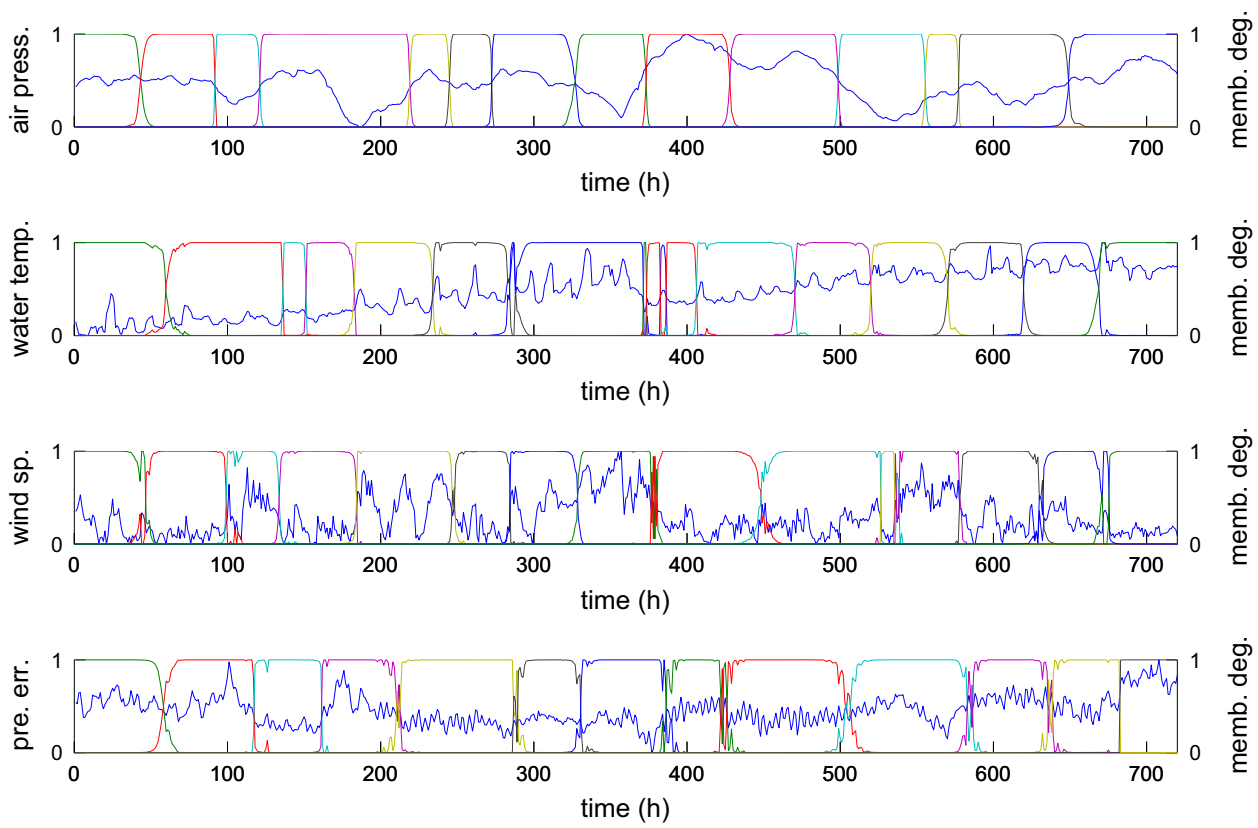

Fig. 9. Optimal membership degrees for different variables (King Point).
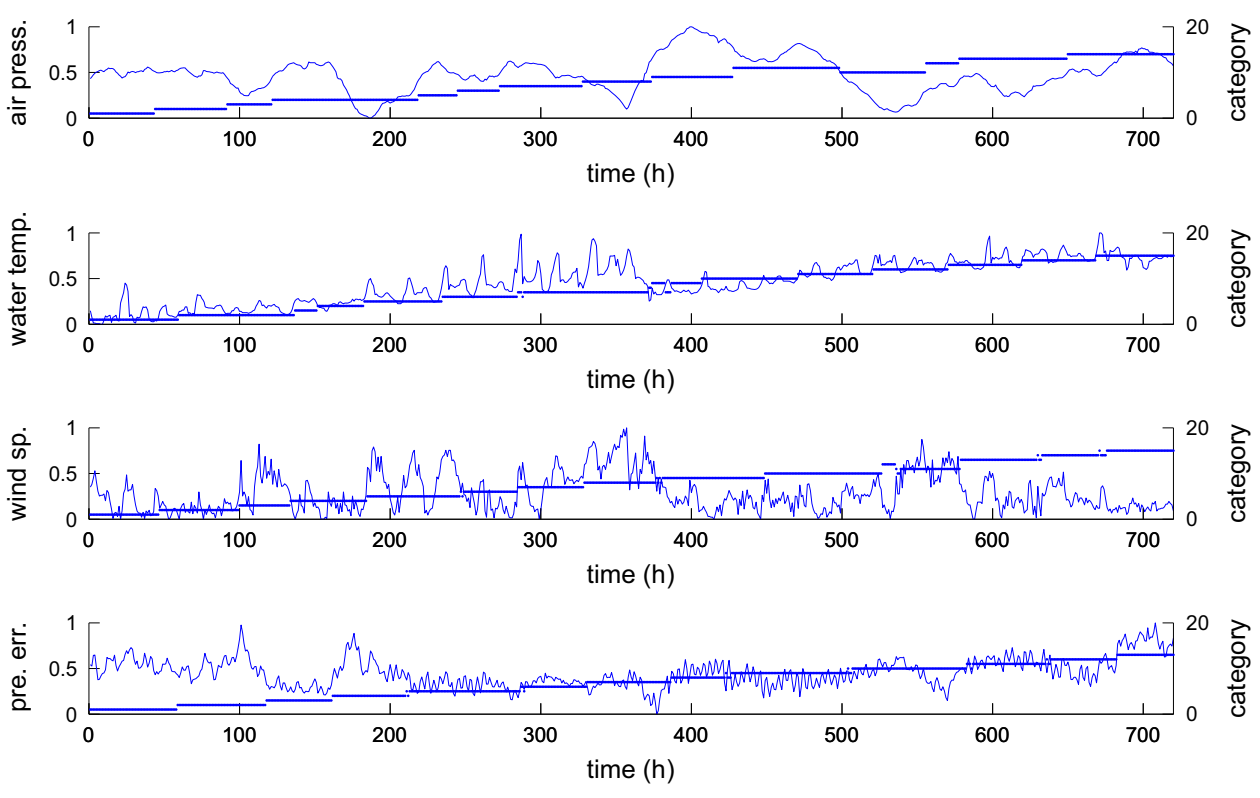

Fig. 10. Crisp segmentation results for different variables (King Point).

Table 1

Segmentation crisp points for the variables (King Point).

\begin{tabular}{ll}
\hline Factors & Change points \\
\hline Air pressure & $1,44,92,122,219,245,273,328,374,428,499,556,578,650$ \\
Water temperature & $1,60,137,152,183,235,285,288,372,374,383,387,407,471,521,571,620,669$ \\
Wind speed & $1,47,100,134,185,247,248,249,285,329,377,380,381,449,527,536,537,539,579,630,631,633,671,672,676$ \\
Residual error & $1,59,118,162,211,212,213,287,289,290,331,387,427,504,505,507,583,586,587,636,637,639,683$ \\
\hline
\end{tabular}

\section{Simulation of tidal level prediction based on modular prediction model}

\subsection{Structure of modular prediction model}

Harmonic method is an efficient and stable tidal level prediction approach which represents the influence of celestial factors.
However, the change of tidal level is a complex process which is affected not only by celestial bodies but also by environmental changes. These complex features make it hard to describe the complex dynamics underlying the tidal changes by a strictly founded model. However, OS-ELM has demonstrated its efficiency in describing nonlinear processes. Taking both advantages of harmonic method and OS-ELM, we present a modular prediction 
Table 2

Sampling pool at different running steps.

\begin{tabular}{|c|c|c|c|c|c|c|c|}
\hline Running step & $1-60$ & $61-92$ & $93-100$ & $101-118$ & 119-137 & $138-162$ & $163-219$ \\
\hline Sampling pool & $1-t$ & $44-t$ & $47-t$ & $59-t$ & $60-t$ & $118-t$ & $122-t$ \\
\hline Running step & $220-235$ & $236-247$ & $248-287$ & $288-328$ & $329-372$ & $373-374$ & $375-377$ \\
\hline Sampling pool & $183-t$ & $185-t$ & $211-t$ & $273-t$ & $285-t$ & $328-t$ & $329-t$ \\
\hline Running step & $378-387$ & $388-428$ & $429-449$ & $450-471$ & $472-504$ & $505-527$ & $528-556$ \\
\hline Sampling pool & $331-t$ & $374-t$ & $377-t$ & $407-t$ & $427-t$ & $449-t$ & $499-t$ \\
\hline Running step & $557-583$ & $584-620$ & $621-650$ & $651-669$ & $670-720$ & & \\
\hline Sampling pool & $507-t$ & $571-t$ & $578-t$ & $620-t$ & $650-t$ & & \\
\hline
\end{tabular}

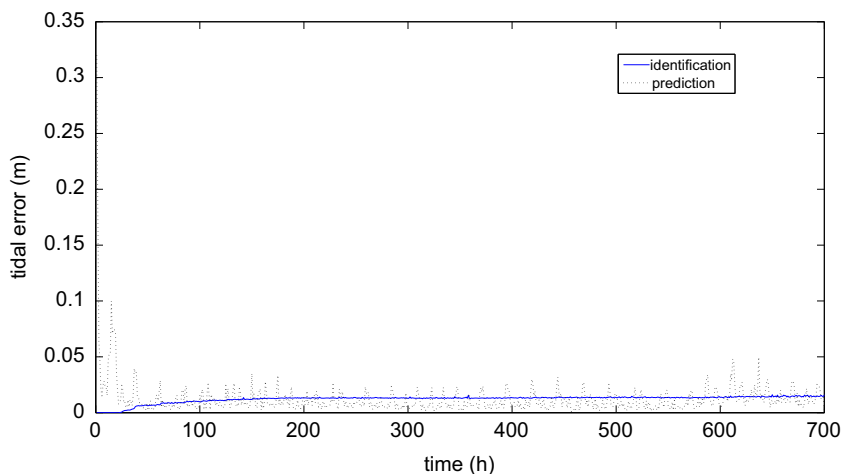

Fig. 11. Identification and prediction error for 1-step-ahead tidal prediction by using OS-ELM based on IGG (King Point).

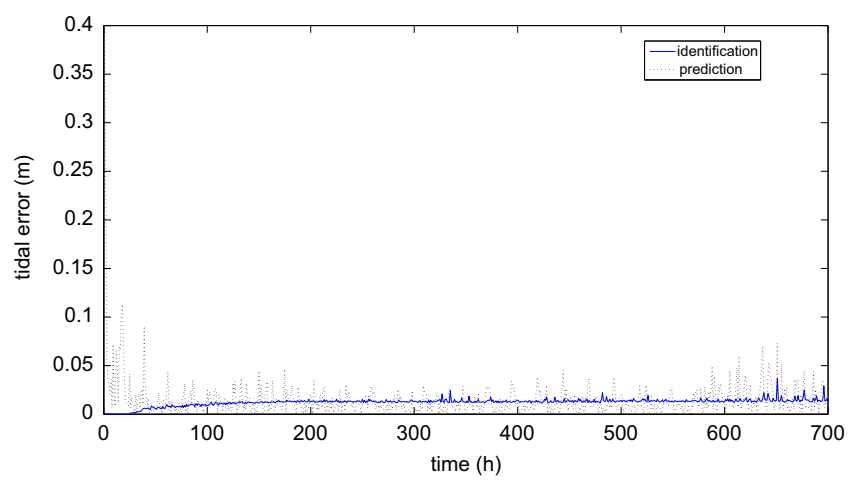

Fig. 12. Identification and prediction error for 1-step-ahead tidal prediction by using conventional OS-ELM (King Point).

Table 3

Simulation results of online ship roll motion prediction.

\begin{tabular}{|c|c|c|c|c|c|c|}
\hline \multirow{2}{*}{$\begin{array}{l}\text { Algorithms } \\
\text { Results }\end{array}$} & \multicolumn{2}{|c|}{ OS-ELM based on IGG } & \multicolumn{4}{|l|}{ OS-ELM } \\
\hline & $R M S E_{\mathrm{I}}(\mathrm{m})$ & $\begin{array}{l}R M S E_{\mathrm{P}} \\
(\mathrm{m})\end{array}$ & Time (s) & $\begin{array}{l}R M S E_{I} \\
(\mathrm{~m})\end{array}$ & $\begin{array}{l}R M S E_{\mathrm{P}} \\
(\mathrm{m})\end{array}$ & $\begin{array}{l}\text { Time } \\
\text { (s) }\end{array}$ \\
\hline 1-h-ahead & 0.0109 & 0.0229 & 0.0085 & 0.0123 & 0.0286 & 0.0057 \\
\hline 3-h-ahead & 0.0206 & 0.0294 & 0.0079 & 0.0592 & 0.0362 & 0.0056 \\
\hline 6-h-ahead & 0.0213 & 0.0373 & 0.0079 & 0.1168 & 0.1515 & 0.0064 \\
\hline $\begin{array}{l}\text { 12-h- } \\
\text { ahead }\end{array}$ & 0.0352 & 0.0401 & 0.0088 & 0.1910 & 0.2314 & 0.0062 \\
\hline
\end{tabular}

scheme, where the conventional harmonic analysis method is utilized to reflect the influences of celestial bodies and shallow water effects, and the proposed IGG-based OS-ELM is implemented to represent the time-varying influences caused by environmental changes. The clarity and stability of the harmonic method

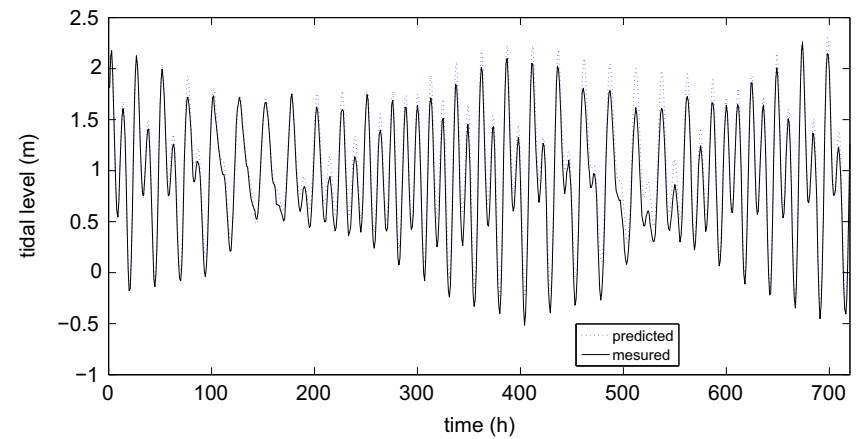

Fig. 13. Measured tidal level and the predicted results by using harmonic method (Mokuoloe).

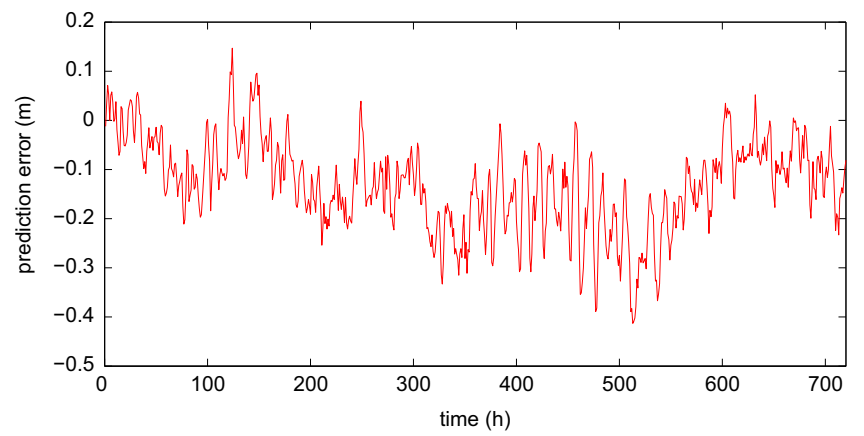

Fig. 14. Prediction error by using harmonic method (Mokuoloe).

and the variability of the proposed IGG-based OS-ELM are combined to achieve higher accuracy on the basis of stable prediction [38].

As the conventionally used tidal prediction method, harmonic method can give stable long-term tidal predictions. Therefore, the harmonic method is employed as the mechanism module in this modular scheme. The result of harmonic prediction is a superposition of constituents whose amplitudes and frequencies are determined by analysis based on the long-term tidal measurements. Thus, the tidal level can be predicted as a time-dependant function which serves as mechanism prediction model:

$Y_{\mathrm{M}}(t)=a_{0}+\sum_{i=1}^{n} h_{i} \cos \left(\omega_{i} t-\phi_{i}\right)+\varepsilon_{i}$

where $a_{0}$ is the height of mean sea level (MSL), $n$ is the number of constituents. $h_{i}, \omega_{i}$ and $\phi_{i}$ are the amplitude, frequency and phase of the corresponding constituent, respectively. And $\varepsilon_{i}$ is the unmolded 

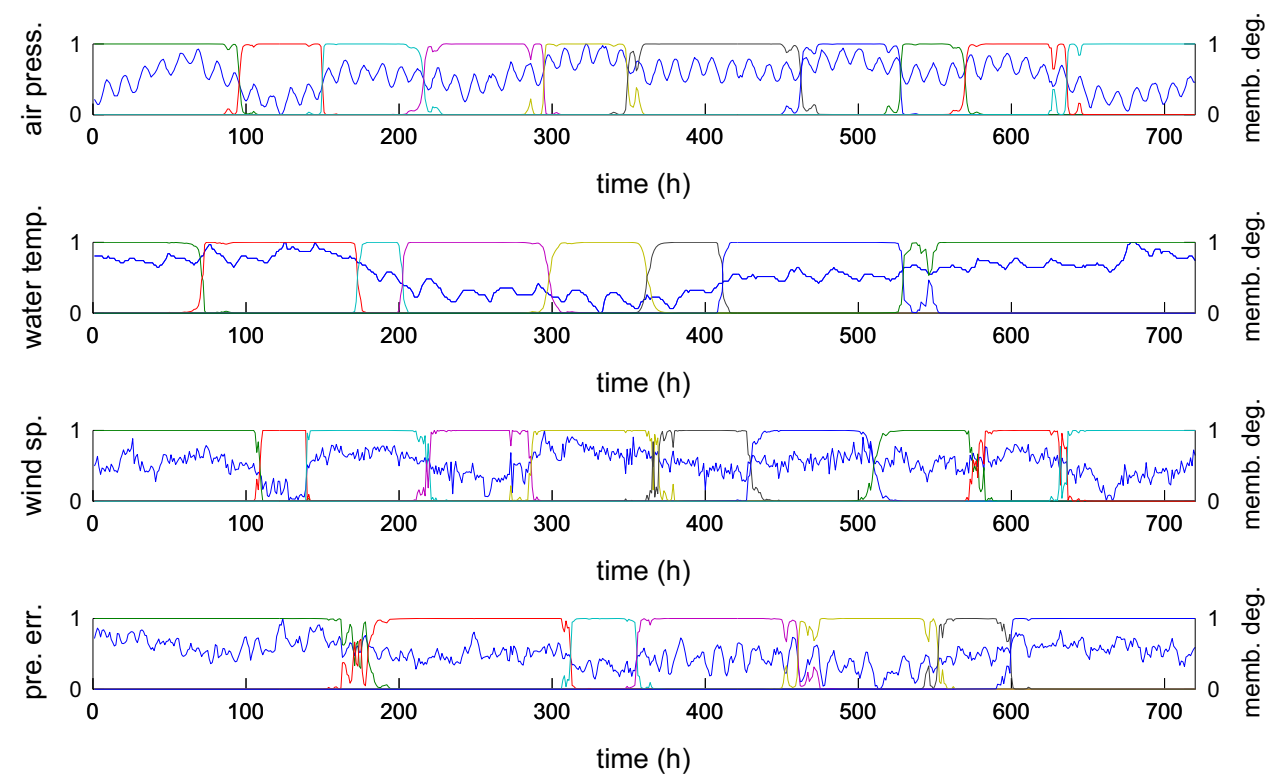

Fig. 15. Optimal membership degrees for different variables (Mokuoloe).
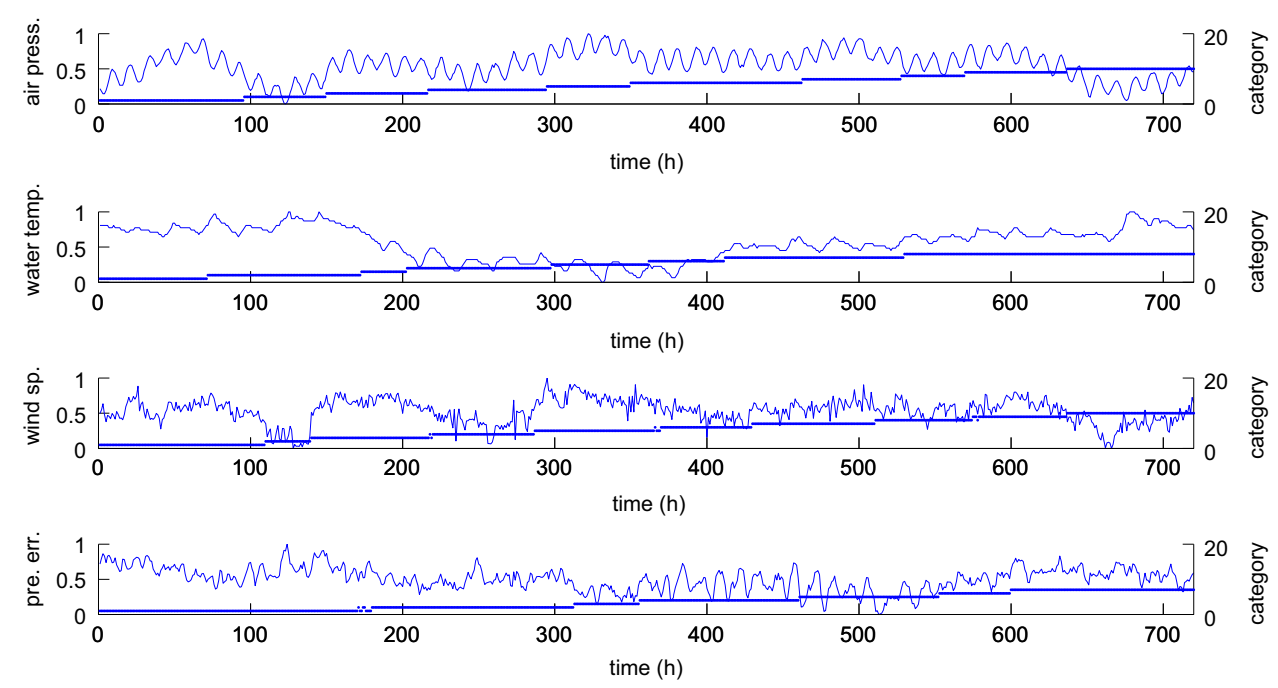

Fig. 16. Crisp segmentation results for different variables (Mokuoloe).

Table 4

Segmentation crisp points for the variables (Mokuoloe).

\begin{tabular}{ll}
\hline Factors & Change points \\
\hline Air pressure & $1,96,150,217,295,350,463,528,570,637$ \\
Water temperature & $1,72,173,203,298,362,412,530$ \\
Wind speed & $1,110,140,218,220,287,366,367,380,430,511,575,578,579,632,633,637$ \\
Residual error & $1,171,172,174,176,180,313,356,461,553,600$ \\
\hline
\end{tabular}

Table 5

Sampling pool at different running steps (Mokuoloe).

\begin{tabular}{|c|c|c|c|c|c|c|c|}
\hline Running step & $1-170$ & $171-172$ & $173-217$ & $218-312$ & $313-365$ & $366-460$ & $461-529$ \\
\hline Sampling pool & $1-t$ & $72-t$ & $140-t$ & $180-t$ & $287-t$ & $356-t$ & $412-t$ \\
\hline Running step & $530-552$ & $553-574$ & $575-720$ & & & & \\
\hline Sampling pool & $461-t$ & $511-t$ & $530-t$ & & & & \\
\hline
\end{tabular}

error which will be modeled by OS-ELM. The number of constituents is determined according to the requirement of accuracy, and higher prediction accuracy can be achieved by employing more constituents.
However, the calculation of larger number of constituents needs more tidal measurements and will involve more computational burden. 
The OS-ELM-based prediction is a kind of sequential learning scheme whose sampling pool is decided online based on IGG in this study. Both processes of identification and prediction are conducted at each step. The configuration of the identification process of the modular model is illustrated as Fig. 2.

It can be noticed in Fig. 2 that two operations are conducted during the identification process. Firstly, the harmonic method is implemented to describe the periodical tidal change which is driven by the revolution of celestial bodies, and its prediction result of tidal level $y$ is denoted as $y_{M}$. $y_{R}$ denotes the residual between measured tidal level $y(t)$ and predicted one $y_{\mathrm{M}}$. The OSELM is used for online tidal prediction of $y_{R}$ based on nonlinear autoregressive with exogenous inputs (NARX) model:

$y_{\mathrm{R}}(t)=f\left(y_{\mathrm{R}}(t-1), \ldots, y_{\mathrm{R}}\left(t-n_{y}\right), u(t-1), \ldots, u\left(t-n_{u}\right)\right)$,

where $y$ and $u$ are system output and input, respectively, with $n_{y}$ and $n_{u}$ being orders of $y$ and $u$, respectively. In this study, $u$ in (36) contains environmental factors including water temperature $T$, air

Table 6

Simulation results of online ship roll motion prediction.

\begin{tabular}{|c|c|c|c|c|c|c|}
\hline \multirow{2}{*}{$\begin{array}{l}\text { Algorithms } \\
\text { Results }\end{array}$} & \multicolumn{2}{|c|}{ OS-ELM based on IGG } & \multicolumn{4}{|l|}{ OS-ELM } \\
\hline & $R M S E_{\mathrm{I}}(\mathrm{m})$ & $\begin{array}{l}R M S E_{\mathrm{P}} \\
(\mathrm{m})\end{array}$ & Time (s) & $\begin{array}{l}R M S E_{1} \\
(\mathrm{~m})\end{array}$ & $\begin{array}{l}R M S E_{\mathrm{P}} \\
(\mathrm{m})\end{array}$ & $\begin{array}{l}\text { Time } \\
\text { (s) }\end{array}$ \\
\hline 1-h-ahead & 0.0141 & 0.0242 & 0.0087 & 0.0157 & 0.0290 & 0.0066 \\
\hline 3-h-ahead & 0.0208 & 0.0571 & 0.0089 & 0.0254 & 0.0628 & 0.0065 \\
\hline 6-h-ahead & 0.0279 & 0.0373 & 0.0087 & 0.0556 & 0.0904 & 0.0070 \\
\hline $\begin{array}{l}\text { 12-h- } \\
\text { ahead }\end{array}$ & 0.0496 & 0.0790 & 0.0088 & 0.1412 & 0.1750 & 0.0072 \\
\hline
\end{tabular}

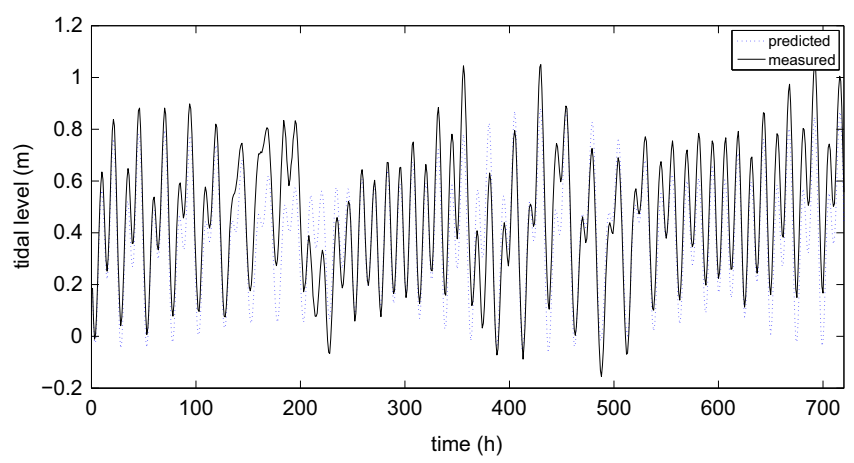

Fig. 17. Measured tidal level and the predicted results by using harmonic method (Old Port Tampa). pressure $P$ and wind speed $V$. That is, $u(t-1), \ldots, u\left(t-n_{u}\right)$ contains $T(t-1), \ldots, T\left(t-n_{T}\right), P(t-1), \ldots, P\left(t-n_{P}\right), V(t-1), \ldots, V\left(t-n_{V}\right)$,

where $n_{T}, n_{P}$ and $n_{V}$ are orders of the $T, P$ and $V$ in prediction model, respectively. The residual information of tidal level $y_{R}$ is considered as the effects of time-varying environmental changes $u$ and other unmodeled factors.

The 1-step-ahead prediction is realized by replacing the $t$ in (36) with $t+1$ :

$y_{\mathrm{R}}(t+1)=f\left(y_{\mathrm{R}}(t), \ldots, y_{\mathrm{R}}\left(t-n_{y}+1\right), u(t), \ldots, u\left(t-n_{u}+1\right)\right)$,

The prediction is then performed by measured variables which is currently available.

For multi-step prediction, for instance $m$-steps-ahead prediction, the processes of identification and prediction are expressed as follows:

$y_{\mathrm{R}}(t)=f\left(y_{\mathrm{R}}(t-m), \ldots, y_{\mathrm{R}}\left(t-m-n_{y}+1\right), u(t-m), \ldots, u\left(t-m-n_{u}+1\right)\right)$,

and

$y_{\mathrm{R}}(t+m)=f\left(y_{\mathrm{R}}(t), \ldots, y_{\mathrm{R}}\left(t-n_{y}+1\right), u(t-m), \ldots, u\left(t-m-n_{u}+1\right)\right)$.

After the OS-ELM is constructed by learning data pairs of $y_{\mathrm{R}}$ in current selected pool, the modular prediction model is achieved by combining the OS-ELM with the harmonic method. The configuration of the prediction process in the modular model is illustrated as Fig. 3.

Once the identification process is completed, currently available information of $y_{\mathrm{R}}, P, T$ and $V$ are then set as input according to $(40)$ and $y_{\mathrm{R}}(t+m)$ is the $m$-steps-ahead prediction of the environment factors' influence to the tidal level.

During the prediction process, predictions generated by the two modules are summed together to achieve the final prediction model. That is, the two identification modules are combined in series connection to form the modular forecast model and get the final tidal prediction result.

\subsection{Real-time tidal prediction simulation}

Online tidal level prediction simulations were conducted to verify the feasibility and efficiency of the proposed sample selection scheme OS-ELM and modular prediction strategy. The simulations employ the measured hourly tidal data of three American Ports of King Point in New York, Mokuoloe in Hawaii and Old Port Tampa in Florida. The hourly samples are measured from GMT0000 April 1 to GMT2300 April 30, 2014, 720 samples in total. All the measurements of tidal level, air pressure, wind speed

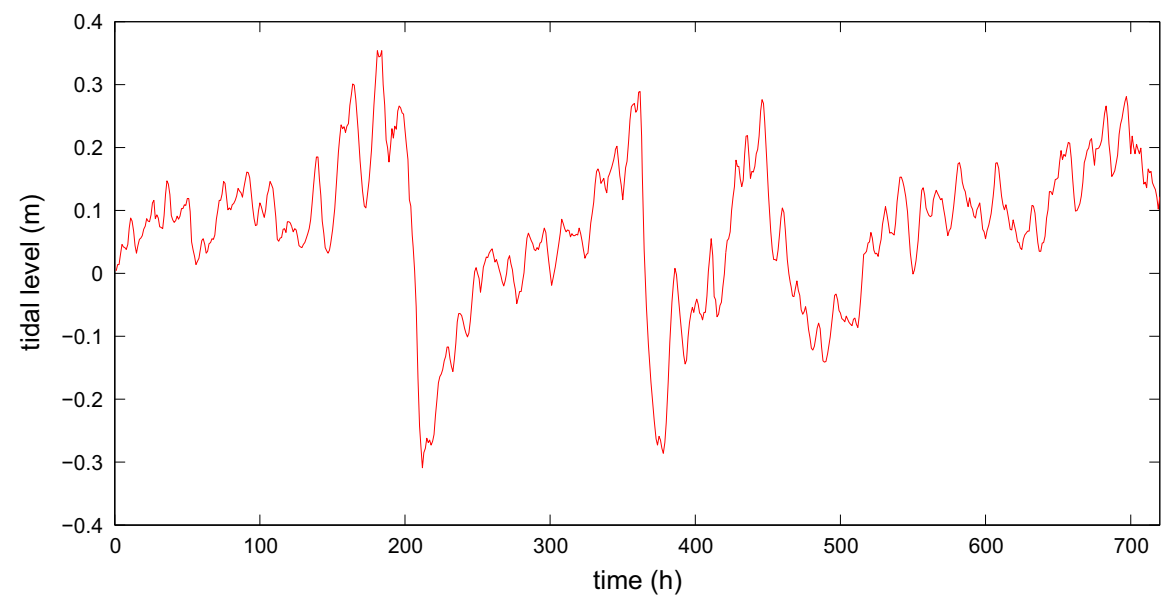

Fig. 18. Prediction error by using harmonic method (Old Port Tampa). 

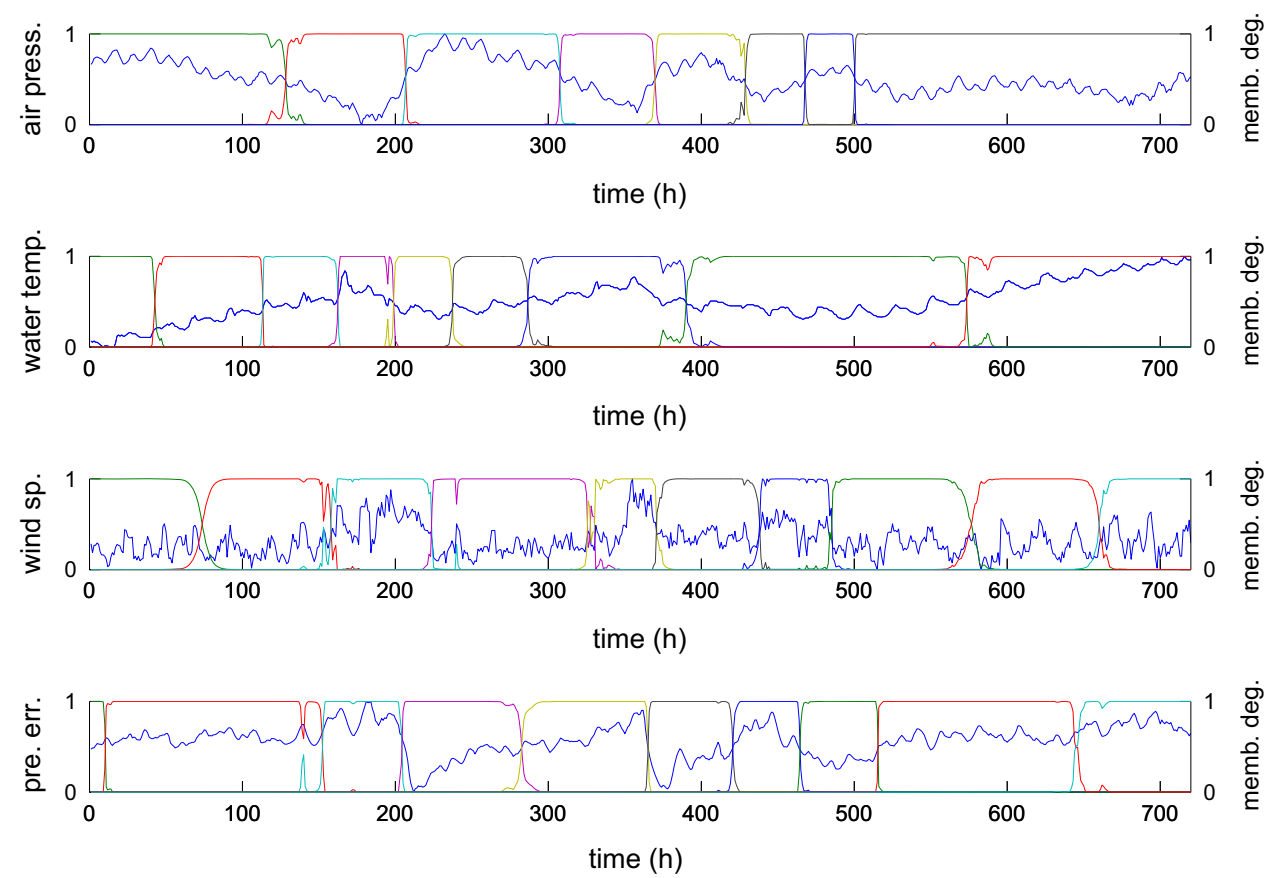

Fig. 19. Optimal membership degrees for different variables (Old Port Tampa).
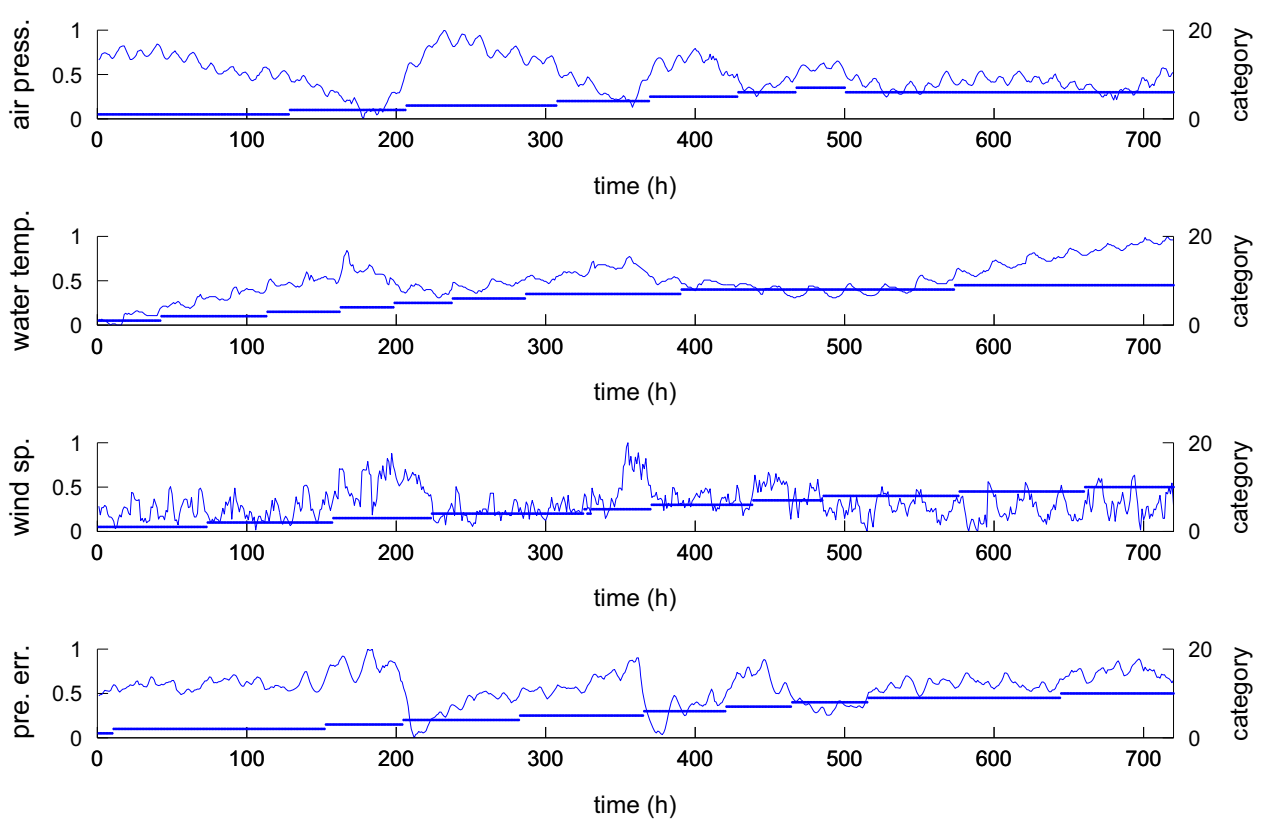

Fig. 20. Crisp segmentation results for different variables (Old Port Tampa).

Table 7

Simulation results of online ship roll motion prediction.

\begin{tabular}{|c|c|c|c|c|c|c|}
\hline \multirow{2}{*}{$\begin{array}{l}\text { Algorithms } \\
\text { Results }\end{array}$} & \multicolumn{2}{|c|}{ OS-ELM based on IGG } & \multicolumn{4}{|l|}{ OS-ELM } \\
\hline & $R M S E_{\mathrm{I}}(\mathrm{m})$ & $\begin{array}{l}R M S E_{\mathrm{P}} \\
(\mathrm{m})\end{array}$ & Time (s) & $\begin{array}{l}R M S E_{\mathrm{I}} \\
(\mathrm{m})\end{array}$ & $\begin{array}{l}R M S E_{\mathrm{P}} \\
(\mathrm{m})\end{array}$ & $\begin{array}{l}\text { Time } \\
\text { (s) }\end{array}$ \\
\hline 1-h-ahead & 0.0117 & 0.0196 & 0.0097 & 0.0131 & 0.0210 & 0.0081 \\
\hline 3-h-ahead & 0.0222 & 0.0304 & 0.0088 & 0.0254 & 0.0460 & 0.0079 \\
\hline 6-h-ahead & 0.0279 & 0.0497 & 0.0102 & 0.0556 & 0.0953 & 0.0086 \\
\hline $\begin{array}{l}\text { 12-h- } \\
\text { ahead }\end{array}$ & 0.0386 & 0.0623 & 0.0091 & 0.1292 & 0.1805 & 0.0076 \\
\hline
\end{tabular}

and water temperature and the parameters of 37 harmonic constants in this study are achieved from web site of American National Oceanic and Atmospheric Administration: http://co-ops. nos.noaa.gov. In the online tidal prediction simulations, both the identification and prediction processes are performed in each step. Simulations are processed in MATLAB 7.4 environment running at $2.40 \mathrm{GHz}$ (CPU) and $1.92 \mathrm{~GB}$ memory (RAM).

The measured tidal data of Port of King Point are shown in Fig. 4. The prediction result achieved by using conventional harmonic method is also shown in the figure. Based on the measured data, the harmonic prediction method is used for tidal prediction and the $R M S E_{\mathrm{P}}$ is $0.181680 \mathrm{~m}$. 
As the harmonic method only takes into consideration the period influences of celestial bodies and ignores the influences of the environmental changes, there exist time-varying errors in the prediction results, as shown in Fig. 4. To depict the time-varying error more clearly, the curve of prediction error by using harmonic method is shown in Fig. 5.

It can be seen from Fig. 5 that the prediction error fluctuates with time and the maximum prediction error reaches nearly $0.7 \mathrm{~m}$. Ship voyage plan stipulated based on such prediction may cause accidents of grounding or collision when ship sails through shallow water or under bridge. Therefore, there is a practical need to reduce predictive tidal error and give accurate predictions. In this study, the index of root mean square error (RMSE) is utilized to evaluate the performances of identification and prediction, respectively:

$R M S E_{\mathrm{I}}=\sqrt{\frac{\sum_{i=1}^{n}\left(y_{i}-y_{\mathrm{I}}\right)^{2}}{n}}$,

$R M S E_{\mathrm{P}}=\sqrt{\frac{\sum_{i=1}^{n}\left(y_{i}-y_{\mathrm{P}}\right)^{2}}{n}}$,

Here $n$ denotes the number of samples, $y_{\mathrm{I}}$ and $y_{\mathrm{P}}$ are the identified and predicted values of $y$, respectively.

The residual prediction error by using harmonic method comprises many useful information thus is considered as predictable here. Therefore, the residual information $y_{R}$ is estimated by OS-ELM based on IGG segmentation in this study. The OS-ELM is adjusted based on the learning of residuals $y_{R}$ and corresponding environmental factors of air pressure $P$, water temperature $T$ and wind speed $V$, as shown in Fig. 6.

It is noticed that the changes of air pressure, water temperature and wind speed are time-varying in nature, which will impose time-varying influences to tidal level changes consequently. To depict current tidal dynamics, we stipulate a time quantum in which the samples can represent the characteristics of current tidal changes. Therefore, we combine the newest time spans corresponding to different input variables as per (34), thus only samples which have close correlation to the current changes are included in the candidate sampling pool. This sample selection strategy helps us to avoid unfavorable effects of samples which have little or contrary effects to current system dynamics, thus guarantee the generalization capability of the OS-ELM.

The fuzzy segmentation is shown in Fig. 7 together with the value of variable normalized to [ -11 ]. It can be seen from Fig. 7 that the proposed algorithm is able to detect meaningful temporal changes of the time series.

The corresponding crisp segmentation is obtained and the results are shown in Fig. 8. The crisp points are considered as changing points corresponding to fluctuations in system dynamics, the time span between two successive crisp points indicates relative stable system dynamics and the samples in the same span are deemed to represent similar tidal characteristics.

The membership degrees for environmental factors $P, T, V$ and $y_{\mathrm{R}}$ are obtained and the results are shown in Fig. 9.

The corresponding crisp segmentation result is shown in Fig. 10. It can be noticed in the figure that some of the crisp changing points of different variables are well coincided. This phenomenon can be further studied to investigate the causal relationship and the latent correlation between changes of system dynamics and relevant environmental factors. During the segmentation, there are 14, 18, 25 and 23 changing points for time series of $P, T, V$ and $y_{\mathrm{R}}$, respectively. The crisp changing point for the 4 variables is listed in Table 1.

During the process of online time series segmentation, there are altogether 26 sampling pools achieved by combining the latest segments of individual variables, and the result of achieved sampling pool is shown in Table 2. In case the sampling pool may be too small under condition of rapid changes of some environmental factors, minimum pool size also sets which enable the stable identification and prediction performance of OS-ELM. The minimum pool size is set as consecutive $25 \mathrm{~h}$ in this study, which covers a whole period of tidal level changes of regular semidiurnal tide. During time spans of 61-69, 138-143, 288-298, 388-399, 584-596, 670-675, the samples in pool size are less than 25 and the minimum pool size takes effect to ensure the stability of the prediction result.

In the study, the running step is set as 700, and the parameters for NARX model are set as $n_{y}=6, n_{P}=1, n_{T}=1$, and $n_{V}=1$. For OS-ELM, the number of hidden neurons assigned to the ELM is 24, and Sigmoid function was selected as activation function. Altogether 50 times of simulation is conducted and the average identification and prediction error of 1-step-ahead tidal level prediction is shown in Fig. 11.

For comparison purpose, the conventional OS-ELM is also conducted and the prediction result is shown in Fig. 12. The samples are presented to OS-ELM sequentially, and the models of identification and prediction are the same as the OS-ELM based on IGG approach.

It is noted by comparing Figs. 11 and 12 that the prediction error has been reduced and the prediction result is more stable with less abrupt changes. Specifically, the predictive RMSE for OS-ELM prediction based on IGG model is $0.0229 \mathrm{~m}$, which is less than $0.0286 \mathrm{~m}$ by using conventional OS-ELM. Both the predictive RMSE of OS-ELM and improved OS-ELM are smaller than $0.1816801 \mathrm{~m}$ by using harmonic method. Simulations of tidal predictions are conducted for more hours ahead prediction and the results are shown in Table 3.

It is shown in Table 3 that, by combining the harmonic predictor and OS-ELM predictor based on IGG, the resulted modular prediction scheme can achieve better generalization accuracy with little loss of processing speed. As the method only needs to process the limited number of samples within the selected sampling pool, it possesses relatively fast processing speed than conventional batch learning methods. The prediction errors of both approaches decrease with the increase of prediction domain, but the proposed approach still remains with relatively high prediction accuracy than that of the conventional OS-ELM method. The simulation results indicate that the optimization of sampling pool for OS-ELM can improve its representing ability for system dynamics especially the dynamics of time-varying dynamics, with tolerable loss to the processing speed of OS-ELM.

To further validate the effectiveness of the proposed method, we adopt the tidal gauges which differ at location and coastal topology. Therefore, the tidal and meteorological measurements in Mokuoloe of Hawaii are also employed. The hourly samples are also measured from GMT0000 April 1 to GMT2300 April 30, 2014. The tidal measurement is depicted in Fig. 13, together with the predicted values predicted via conventional harmonic method.

For comparison purpose, the predicted error by using conventional harmonic method is depicted in Fig. 14.

The optimal membership degrees for environmental factors of $P, T, V$ and $y_{\mathrm{R}}$ are shown in Fig. 15 .

The corresponding optimal crisp segmentation is depicted in Fig. 16 and the sampling pool can be decided accordingly.

It can be noticed in Fig. 16 that during the segmentation, there are $10,8,17$ and 11 changing points for time series of $P, T, V$ and $y_{R}$, respectively. The crisp changing points are listed in Table 4.

The sampling pools for different running steps are achieved based on the crisp segmentation points and the result is shown in Table 5 . The minimum pool size of 25 takes effect only in time span of 366-381.

In the study, the preset simulation settings are the same as that for Port of King Point. That is, the running step is 700, $n_{y}=6$, $n_{P}=1, n_{T}=1$, and $n_{V}=1$ for the NARX predictive model. The number of hidden neurons assigned to the ELM is 24 for OS-ELM, and Sigmoid function was selected as activation function. 
Simulations of tidal predictions are conducted for more hours ahead prediction and the results are shown in Table 6.

Another selected tidal station is Old Port Tampa in Florida, USA. The measurements of tidal level during time span of GMT0000 April 1 to GMT2300 April 30, 2014 are depicted in Fig. 17.

And the predicted error by using conventional harmonic method is depicted in Fig. 18. This is also the residual information used to train the improved OS-ELM.

The optimal membership degrees for environmental factors $P$, $T, V$ and $y_{\mathrm{R}}$ are shown in Fig. 19.

The corresponding optimal crisp segmentation is depicted in Fig. 20.

We skip the detailed statement selection of sampling pool and illustration of identification and prediction performance. The prediction performance is listed in Table 7.

It can be noticed from the above simulation results that the improved OS-ELM performs superior to conventional OS-ELM both in identification and prediction. This can attribute to the sampling selection strategy performed by improved Gath-Geva fuzzy segmentation approach. By selecting samples consciously, the number of the samples to be learned is reduced, and the characteristics of the samples are homogeneous. This enables the precise identification and prediction of time series as well as the fast data processing speed. The modular prediction strategy is implemented in this study, which employ the harmonic method and OS-ELM to represent the influences of celestial bodies' movement and the environmental factors, respectively. This modular strategy takes both advantages of mechanism model performed by harmonic method, and identification model performed by improved OS-ELM. It makes use of conventional harmonic method to generate stable long-term prediction, and the improved OS-ELM only needs to focus on the extra influences on the tidal changes to improve the prediction accuracy.

\section{Conclusions}

A OS-ELM is proposed based on improved Gath-Geva fuzzy segmentation approach. The approach selects sampling pool consciously and improves the generalization performance of OS-ELM with little loss of processing speed. The sampling pool exhibits better realtime representing ability for time-varying system dynamics. Simulations are conducted to validate the effectiveness of the proposed approach, with the achieved OS-ELM being performed as a module for representing influences of environmental factors. The OS-ELM module is combined with conventional harmonic method and the resulted modular prediction scheme possesses satisfying accuracy, prediction stability and processing speed. The novel sampling pool selection can also be implemented to system analysis and other occasions of online identification and prediction applications for time-varying systems.

\section{Acknowledgments}

This work is supported by National Natural Science Foundation of China (Grant no. 51279106), the Applied Basic Research Fund of the Chinese Ministry of Transport (Grant no. 2014329225010) and Fundamental Research Funds for the Central Universities (Grant no. 3132014028).

\section{References}

[1] G.H. Fang, W.Z. Zheng, Z.Y. Chen, Analysis and Prediction of Tide and Tidal Current, Ocean Press, Beijing, 1986.

[2] T. Lee, Back-propagation neural network for long-term tidal predictions, Ocean Eng. 31 (2004) 225-238.

[3] S. Liang, M. Li, Z. Sun, Prediction models for tidal level including strong meteorologic effects using a neural network, Ocean Eng. 35 (2008) 666-675.
[4] I. Malekmohamadi, M. Bazargan-Lar, R. Kerachian, et al., Evaluating the efficacy of SVMs, BNs, ANNs and ANFIS in wave height prediction, Ocean Eng. 38 (2011) 487-497.

[5] S. Haykin, Neural Networks: A Comprehensive Foundation, Prentice Hall, NJ, 1999.

[6] T. Lee, D. Jeng, Application of artificial neural networks in tide-forecasting, Ocean Eng. 29 (2002) 1003-1022.

[7] J.C. Yin, Z.J. Zou, F. Xu, Sequential learning radial basis function network for real-time tidal level predictions, Ocean Eng. 57 (2013) 49-55.

[8] J. Platt, A resource allocating network for function interpolation, Neural Comput. 3 (2) (1991) 213-225.

[9] Y.W. Lu, N. Sundararajan, P. Saratchandran, A sequential learning scheme for function approximation using minimal radial basis function neural networks, Neural Comput. 9 (1997) 461-478.

[10] S. Suresh, K. Dong, H. Kim, A sequential learning algorithm for self-adaptive resource allocation network classifier, Neurocomputing 7 (3) (2010) 3012-3019.

[11] J.C. Yin, G.S. Li, J.Q. Hu, A modular prediction mechanism based on sequential extreme learning machine with application to real-time tidal prediction, in: Extreme Learning Machines 2013: Algorithms and Applications, vol. 16, 2013. pp. 35-54.

[12] J.M. Li, X.F. Chen, Z.J. He, Adaptive stochastic resonance method for impact signal detection based on sliding window, Mech. Syst. Signal Process. 36 (2013) 240-255.

[13] V.A. Akpan, G.D. Hassapis, Nonlinear model identification and adaptive model predictive control using neural networks, ISA Trans. 50 (2011) 177-194.

[14] N.Y. Liang, G.B. Huang, P. Saratchandran, N. Sundararajan, A fast and accurate online sequential learning algorithm for feedforward networks, IEEE Trans. Neural Netw. 17 (6) (2006) 1411-1423.

[15] G.B. Huang, Q.Y. Zhu, C.K. Siew, Extreme learning machine: theory and applications, Neurocomputing 70 (2006) 489-501.

[16] G.B. Huang, D.H. Wang, Y. Lan, Extreme learning machines: a survey, Int. J. Mach. Learn. Cybern. 2 (2) (2011) 107-122.

[17] A. Kehagias, A hidden Markov model segmentation procedure for hydrological and environmental time series, Stoch. Environ. Res. Risk Assess. 18 (2) (2004) 117-130.

[18] P. Hubert, The segmentation procedure as a tool for discrete modeling of hydrometeorological regimes, Stoch. Environ. Res. Risk Assess. 14 (4-5) (2000) 297-304.

[19] A. Kehagias, E. Nidelkou, V. Petridis, A dynamic programming segmentation procedure for hydrological and environmental time series, Stoch. Environ. Res. Risk Assess. 20 (2005) 77-94.

[20] H. Aksoy, Hydrological variability of the European part of Turkey, Iran. J. Sci. Technol. 31 (B2) (2007) 225-236.

[21] H. Aksoy, A. Gedikli, N.E. Unal, et al., Fast segmentation algorithms for long hydrometeorological time series, Hydrol. Process. 22 (23) (2008) 4600-4608.

[22] A. Gedikli, H. Aksoy, N.E. Unal, Segmentation algorithm for long time series analysis, Stoch. Environ. Res. Risk Assess. 22 (3) (2008) 291-302.

[23] A. Gedikli, H. Aksoy, N.E. Unal, A. Kehagias, Modified dynamic programming approach for offline segmentation of long hydrometeorological time series, Stoch. Environ. Res. Risk Assess. 24 (2010) 547-557.

[24] A. Gedikli, H. Aksoy, AUG-Segmenter: a user-friendly tool for segmentation of long time series, J. Hydroinformatics 12 (3) (2010) 318-328.

[25] J. Abonyi, B. Feil, S. Nemeth, P. Arva, Modified Gath-Geva clustering for fuzzy segmentation of multivariate time-series, Fuzzy Sets Syst. 149 (2005) 39-56.

[26] I. Gath, A.B. Geva, Unsupervised optimal fuzzy clustering, IEEE Trans. Pattern Anal. Mach. Intell. 7 (1989) 773-780.

[27] J. Abonyi, B. Feil, S. Nemeth, P. Arva, Fuzzy clustering based segmentation of timeseries, in: Lecture Notes in Computer Science, vol. 2810, 2003, pp. 275-286.

[28] N.N. Wang, X.D. Liu, J.C. Yin, Improved Gath-Geva clustering for fuzzy segmentation of hydrometeorological time series, Stoch. Environ. Res. Risk. Assess. 26 (1) (2012) 139-155.

[29] M. Figueiredo, A.K. Jain, Unsupervised learning of finite mixture models, IEEE Trans. Pattern Anal. Mach. Intell. 24 (3) (2002) 381-396.

[30] G. Celeux, S. Chretien, F. Forbes, A. Mkhadri, A Component-wise EM Algorithm for Mixtures, Technical Report 3746, INRIA, France, 1999.

[31] N. Jiang, Z.Y. Zhao, L.Q. Ren, Design of structural modular neural networks with genetic algorithm, Adv. Eng. Softw. 34 (2003) 17-24.

[32] G.B. Huang, X.J. Ding, H.M. Zhou, Optimization method based extreme learning machine for classification, Neurocomputing 74 (2010) 155-163.

[33] G.B. Huang, H.M. Zhou, X.J. Ding, R. Zhang, Extreme learning machine for regression and multiclass classification, IEEE Trans. Syst. Man Cybern. B 42 (2) (2012) 513-529.

[34] R. Povinelli, M. Johnson, A. Lindgren, J. Ye, Time series classification using Gaussian mixture models of reconstructed phase spaces, IEEE Trans. Knowl. Data Eng. 16 (6) (2004) 779-783.

[35] H. Vernieuwe, B. De Baets, N.E.C. Verhoest, Comparison of clustering algorithms in the identification of Takagi-Sugeno models: a hydrological case study, Fuzzy Sets Syst. 157 (2006) 2876-2896.

[36] E.I. Athanasiadis, D.A. Cavouras, P.P. Spyridonos, D.T. Glotsos, I.K. Kalatzis G.C. Nikiforidis, Complementary DNA microarray image processing based on the fuzzy Gaussian mixture model, IEEE Trans. Inf. Technol. Biomed. 13 (4) (2009) 419-425.

[37] Z. Fu, A. Robles-Kelly, J. Zhou, Mixing linear SVMs for nonlinear classification, IEEE Trans. Neural Netw. 21 (2010) 1963-1975.

[38] M.J. Mizianty, L. Kurgan, Modular prediction of protein structural classes from sequences of twilight-zone identity with predicting sequences, BMC Bioinform. 10 (2009) 414-437. 


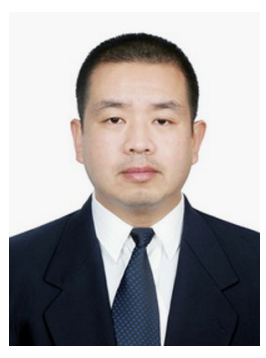

Jian-Chuan Yin was born in Shandong Province, China. He received his Ph.D. degree in Traffic Information Engineering from Dalian Maritime University, Dalian, China, in 2007. He joined the Navigation College, Dalian Maritime University in 2008, where he is currently an associate professor. From 2010 to 2012, he was a post doctoral fellow with the School of Naval Architecture, Ocean and Civil Engineering, Shanghai Jiao Tong University, Shanghai, China. His research interests include ocean engineering, computational intelligence and ship control.

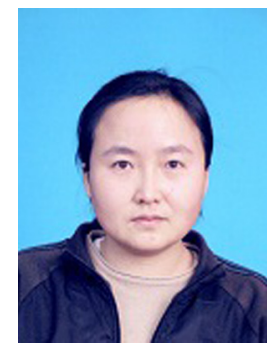

Ni-Ni Wang received her Ph. D. degree in Contro Theory and Control Engineering from Dalian University of Technology, Dalian, China. She is now a post doctoral fellow with the Institute of Geographic Science and Natural Resources Research, Chinese Academy of Sciences, Beijing, China. Her research interests include neural networks, fuzzy sets and their applications. 